Algebraic $8 \mathcal{G}$ Geometric $\mathcal{T}_{\text {opology }}$

Volume 4 (2004) 893-934

Published: 13 October 2004

\title{
ATG
}

\section{Eta invariants as sliceness obstructions and their relation to Casson-Gordon invariants}

\author{
STEFAN FRIEDL
}

\begin{abstract}
We give a useful classification of the metabelian unitary representations of $\pi_{1}\left(M_{K}\right)$, where $M_{K}$ is the result of zero-surgery along a knot $K \subset S^{3}$. We show that certain eta invariants associated to metabelian representations $\pi_{1}\left(M_{K}\right) \rightarrow U(k)$ vanish for slice knots and that even more eta invariants vanish for ribbon knots and doubly slice knots. We show that our vanishing results contain the Casson-Gordon sliceness obstruction. In many cases eta invariants can be easily computed for satellite knots. We use this to study the relation between the eta invariant sliceness obstruction, the eta-invariant ribbonness obstruction, and the $L^{2}$-eta invariant sliceness obstruction recently introduced by Cochran, Orr and Teichner. In particular we give an example of a knot which has zero eta invariant and zero metabelian $L^{2}$-eta invariant sliceness obstruction but which is not ribbon.
\end{abstract}

AMS Classification 57M25, 57M27; 57Q45, 57Q60

Keywords Knot concordance, Casson-Gordon invariants, Eta invariant

\section{Introduction}

\subsection{A quick trip through knot concordance theory}

A knot $K \subset S^{n+2}$ is a smooth oriented submanifold homeomorphic to $S^{n}$. A knot $K$ is called slice if it bounds a smooth $(n+1)$-disk in $D^{n+3}$. Isotopy classes of knots form a semigroup under connected sum. The quotient of this semigroup by the subsemigroup of slice knots turns out to be a group, called the knot concordance group. For example, the existence of inverses follows by noting that the connected sum of $K$ and $-r K$ bounds a disk, where $-r K$ denotes the knot obtained by reflecting $K$ through a disjoint hypersphere and reversing the orientation. It is a natural goal to attempt to understand this group and to find complete invariants for detecting when a knot is slice.

Knot concordance in the high-dimensional case is well understood. For even $n$ Kervaire [Ker65] showed that every knot $K \subset S^{n+2}$ is slice, and for odd $n \geq 3$ 
Levine [Lev69] showed that $K \subset S^{n+2}$ is slice if and only if $K$ is algebraically slice (cf. section 2.2 for a definition). In this way, the task of detecting slice knots in higher dimensions was reduced to an algebraic problem which is wellunderstood Lev69b.

The classical case $n=1$ turns out to be a much more difficult problem. Casson and Gordon CG78, CG86] defined certain sliceness obstructions (cf. section 5.1) and used these to give examples of knots in $S^{3}$ which are algebraically slice but not geometrically slice.

Letsche [Let00] introduced two new approaches to finding obstructions to the sliceness of a knot. One approach used the concept of a universal group to find representations that extend over the complement of a slice disk, the other used eta invariants of metabelian representations of knot complements to give sliceness obstructions. The idea of universal groups was taken much further in a ground breaking paper by Cochran, Orr and Teichner [COT03. (cf. section 6). The goal of this paper is to build on Letsche's second approach.

\subsection{Summary of results}

Given a closed smooth three-manifold $M$ and given a unitary representation $\alpha: \pi_{1}(M) \rightarrow U(k)$ Atiyah-Patodi-Singer APS75] defined $\eta(M, \alpha) \in \mathbf{R}$, called the eta invariant of $(M, \alpha)$. This invariant is closely related to signatures, and therefore well-suited for studying cobordism problems. For a knot $K$ we study the eta invariants associated to the closed manifold $M_{K}$, the result of zero-framed surgery along $K \subset S^{3}$.

In proposition 4.1 we give a complete classification of irreducible, unitary, metabelian (cf. section 4) representations of $\pi_{1}\left(M_{K}\right)$. In theorem 4.5 we show that for a slice knot the eta invariant vanishes for certain irreducible metabelian representations of prime power dimensions.

In section 5.1 we recall the Casson-Gordon sliceness obstruction theorem. We show in theorem [5.5 that for a given knot $K$ the Casson-Gordon sliceness obstruction vanishes if and only if $K$ satisfies the vanishing conclusion of theorem 4.5 .

Despite this equivalence of obstructions the eta invariant approach has several advantages over the Casson-Gordon approach. For example we show in theorem 4.7 that eta invariants vanish for tensor products of certain irreducible prime power dimensional representations. This gives sliceness obstructions, which are potentially stronger than the Casson-Gordon obstruction. 
Recently Cochran, Orr and Teichner COT03, COT04 defined the notion of $(n)$-solvability for a knot, $n \in \frac{1}{2} \mathbb{N}$, which has in particular the following properties.

(1) Slice knots are $(n)$-solvable for all $n$.

(2) (n)-solvable knots are $(m)$-solvable for all $m \leq n$.

(3) A knot is (0.5)-solvable if and only if it is algebraically slice.

(4) (1.5)-solvable knots have zero Casson-Gordon obstruction.

Given a homomorphism $\varphi: \pi_{1}(M) \rightarrow G$ to a group $G$, Cheeger and Gromov defined the $L^{2}$-eta invariant $\eta^{(2)}(M, \varphi) \in \mathbf{R}$ which is closely related to Atiyah's $L^{2}$-signature. Cochran, Orr and Teichner used $L^{2}$-eta invariants to find examples of knots which are (2.0)-solvable, which in particular have zero Casson-Gordon obstructions, but which are not (2.5)-solvable. Using similar ideas Taehee Kim Kim02 found examples of knots which are (1.0)-solvable and have zero Casson-Gordon obstructions, but which are not (1.5)-solvable. A quick summary of this theory is given in section [ 6]

In section 17 we give examples that show that $L^{2}$-eta invariants are not complete (non-torsion) invariants for a knot to be (0.5)-solvable respectively (1.5)solvable. In our examples we use a satellite construction to get knots whose eta invariants can be computed explicitly by methods introduced by Litherland Lit84.

The systematic study of eta invariants corresponding to metabelian representations also allows us to find more refined obstructions to a knot being ribbon (theorem 8.3). It is not known whether all slice knots satisfy the ribbon obstruction theorem. The fact that the ribbon obstruction is apparently stronger than the corresponding sliceness obstruction is particularly interesting as this could potentially provide a way to disprove the ribbon conjecture (that every slice knot is ribbon). In proposition 8.9 we give examples of knots for which all abelian and metabelian sliceness obstructions vanish, but which do not satisfy the condition for theorem 8.3, i.e. which are not ribbon. It is not known whether these examples are slice or not.

We also apply our methods to the study of doubly slice knots (theorem 8.4). It is a well-known fact that the doubly sliceness condition is much stronger than the sliceness condition (cf. [S71, Kim03, Kim04). We point out the interesting fact that doubly slice knots satisfy the ribbon obstruction theorem, which suggests that doubly slice knots have a 'higher chance' of being ribbon than ordinary slice knots. 
We conclude this paper with a discussion of Gilmer's Gi83 Gi93 and Letsche's Let00] obstruction theorems in sections 8.3 and 8.4. During our work on eta invariants as sliceness obstructions we found that both theorems have gaps in their proofs. We explain where the problem lies and we show that Gilmer's and Letsche's approach still give ribbonness obstruction. These ribbon obstructions can be shown to be contained in theorem 8.3 .

This paper is essentially the author's thesis. I would like to express my deepest gratitude towards my advisor Jerry Levine for his patience, help and encouragement.

\section{Basic knot theory and linking pairings}

Throughout this paper we will always work in the smooth category. In the classical dimension the theory of knots in the smooth category is equivalent to the theory in the locally flat category. All homology groups are furthermore taken over $\mathbb{Z}$, unless otherwise indicated.

\section{$2.1 \quad$ Basic knot theory}

By a knot $K$ we understand an oriented submanifold of $S^{3}$ diffeomorphic to $S^{1}$. A oriented surface $F \subset S^{3}$ with $\partial(F)=K$ will be called a Seifert surface for $K$. Note that a Seifert surface inherits an orientation from $K$, in particular the map $H_{1}(F) \rightarrow H_{1}\left(S^{3} \backslash F\right), a \mapsto a_{+}$induced by pushing into the positive normal direction is well-defined. The pairing

$$
\begin{aligned}
H_{1}(F) \times H_{1}(F) & \rightarrow \mathbb{Z} \\
(a, b) & \mapsto \operatorname{lk}\left(a, b_{+}\right)
\end{aligned}
$$

is called the Seifert pairing of $F$. Any matrix $A$ representing such a pairing is called a Seifert matrix for $K$. By [Mu65, p. 393], Lev70 two Seifert matrices for a given knot $K$ are S-equivalent. In particular the Alexander polynomial $\Delta_{K}(t):=\operatorname{det}\left(A t-A^{t}\right) \in \mathbb{Z}\left[t, t^{-1}\right]$ is a well-defined invariant of $K$, i.e. independent of the choice of $A$, up to multiplication by units $\pm t^{l}$.

Let $N(K)$ be a solid torus neighborhood of $K$. A meridian $\mu$ of $K$ is a nonseparating simple closed curve in $\partial(N(K))$ that bounds a disc in $N(K)$. The notion of meridian is well-defined up to homotopy in $S^{3} \backslash K$. Alexander duality shows that there exists an isomorphism $\epsilon: H_{1}\left(S^{3} \backslash K\right) \rightarrow \mathbb{Z}$ such that $\epsilon(\mu)=1$. 
We will mostly study invariants of $M_{K}$, the result of zero-framed-surgery along $K$. This manifold has the advantage over $S^{3} \backslash K$ that it is a closed manifold associated to $K$. Note that we have isomorphisms $H_{1}\left(M_{K}\right) \stackrel{\cong}{\leftrightarrows} H_{1}\left(S^{3} \backslash K\right) \stackrel{\epsilon}{\rightarrow} \mathbb{Z}$, we will denote this composition of maps by $\epsilon$ as well.

\section{$2.2 \quad$ Slice knots}

We begin with a few definitions. We say that two knots $K_{1}, K_{2}$ are smoothly concordant if there exists a smooth submanifold $V \subset S^{3} \times[0,1]$ such that $V \cong S^{1} \times[0,1]$ and such that $\partial(V)=K_{0} \times 0 \cup-K_{1} \times 1$. A knot $K \subset S^{3}$ is called smoothly slice if it is concordant to the unknot. Equivalently, a knot is smoothly slice if there exists a smooth disk $D \subset D^{4}$ such that $\partial(D)=K$.

A knot $K$ is called topologically slice if $K$ bounds a locally flat disk $D \subset D^{4}$. Locally flat means that one can find an embedding $D \times D^{2} \subset D^{4}$ which extends the embedding of the disk.

We say that the Seifert pairing on $H_{1}(F)$ is metabolic if there exists a subspace $H$ of half-rank such that the Seifert pairing vanishes on $H$. This is the case if and only if $K$ has a metabolic Seifert matrix $A$, i.e. if $A$ is of the form

$$
A=\left(\begin{array}{cc}
0 & B \\
C & D
\end{array}\right),
$$

where $0, B, C, D$ are square matrices. If the Seifert pairing of a knot is metabolic then we say that $K$ is algebraically slice.

By Levine [Lev69] we now get the following implications

$$
\text { smoothly slice } \Rightarrow \text { topologically slice } \Rightarrow \text { algebraically slice. }
$$

The reverse implications do not hold. Casson and Gordon [CG86] gave examples of algebraically slice knots which are not topologically slice. Freedman FQ90 showed that any knot with trivial Alexander polynomial is topologically slice, whereas Gompf Gom86 gave examples of knots with trivial Alexander polynomial that are not smoothly slice.

In the following we will work in the smooth category. In particular by slice we will mean smoothly slice.

For a slice disk $D$ we define $N_{D}:=\overline{D^{4} \backslash N(D)}$, where $N(D)$ is a tubular neighborhood of $D$ in $D^{4}$. We summarize a couple of well-known facts about $N_{D}$.

(1) $\partial\left(N_{D}\right)=M_{K}$, 
(2) $H_{*}\left(N_{D}\right)=H_{*}\left(S^{1}\right)$,

(3) the inclusion map $H_{1}\left(M_{K}\right) \rightarrow H_{1}\left(N_{D}\right)$ is an isomorphism.

We will denote the induced map $H_{1}\left(N_{D}\right) \cong H_{1}\left(M_{K}\right) \stackrel{\epsilon}{\rightarrow} \mathbb{Z}$ by $\epsilon$ as well.

\subsection{Universal abelian cover of $M_{K}$ and the Blanchfield pairing}

Let $K$ be a knot. Denote the infinite cyclic cover of $M_{K}$ corresponding to $\epsilon: H_{1}\left(M_{K}\right) \rightarrow \mathbb{Z}$ by $\tilde{M}_{K}$. Then $\mathbb{Z}=\langle t\rangle$ acts on $\tilde{M}_{K}$, therefore $H_{1}\left(\tilde{M}_{K}\right)$ carries a $\Lambda:=\mathbb{Z}\left[t, t^{-1}\right]$-module structure. Clearly $H_{1}\left(\tilde{M}_{K}\right)$ and the twisted homology module $H_{1}\left(M_{K}, \Lambda\right)$ are canonically isomorphic as $\Lambda$-modules.

In the following we will give $\Lambda$ the involution induced by $\bar{t}=t^{-1}$. Let $S:=$ $\{f \in \Lambda \mid f(1)=1\}$. The $\Lambda$-module $H_{1}\left(M_{K}, \Lambda\right)$ is $S$-torsion since for example the Alexander polynomial $\Delta_{K}(t)$ lies in $S$ and $\Delta_{K}(t)$ annihilates $H_{1}\left(M_{K}, \Lambda\right)$. Blanchfield [B57] introduced the pairing

$$
\begin{aligned}
\lambda_{B l}: H_{1}\left(M_{K}, \Lambda\right) \times H_{1}\left(M_{K}, \Lambda\right) & \rightarrow S^{-1} \Lambda / \Lambda \\
(a, b) & \mapsto \frac{1}{p(t)} \sum_{i=-\infty}^{\infty}\left(a \cdot t^{i} c\right) t^{-i}
\end{aligned}
$$

where $c \in C_{2}\left(M_{K}, \Lambda\right)$ such that $\partial(c)=p(t) b$ for some $p(t) \in S$. It is well-defined, non-singular and hermitian over $\Lambda$. For any $\Lambda$-submodule $P \subset$ $H_{1}\left(M_{K}, \Lambda\right)$ define

$$
P^{\perp}:=\left\{v \in H_{1}\left(M_{K}, \Lambda\right) \mid \lambda_{B l}(v, w)=0 \text { for all } w \in P\right\} .
$$

If $P \subset H_{1}(M, \Lambda)$ is such that $P=P^{\perp}$ then we say that $P$ is a metabolizer for $\lambda_{B l}$. If $\lambda_{B l}$ has a metabolizer we say that $\lambda_{B l}$ is metabolic.

For a $\mathbb{Z}$-module $A$ denote by $T A$ the $\mathbb{Z}$-torsion submodule of $A$ and let $F A:=$ $A / T A$.

Theorem 2.1 ([Kea75] Let00, prop. 2.8])

(1) If $K$ is slice and $D$ any slice disk, then

$$
P:=\operatorname{Ker}\left\{H_{1}\left(M_{K}, \Lambda\right) \rightarrow F H_{1}\left(N_{D}, \Lambda\right)\right\}
$$

is a metabolizer for the Blanchfield pairing.

(2) The Blanchfield pairing for a knot $K$ is metabolic if and only if $K$ is algebraically slice.

It is an open problem whether every slice knot $K$ admits a slice disk $D$ such that the submodule $\operatorname{Ker}\left\{H_{1}\left(M_{K}, \Lambda\right) \rightarrow H_{1}\left(N_{D}, \Lambda\right)\right\}$ is a metabolizer for the Blanchfield pairing. This is an important question since it is closely linked to the ribbon conjecture (cf. 8.1). 


\subsection{Finite cyclic covers and linking pairings}

Let $K$ be a knot, $k$ some number. Denote by $M_{k}$ the $k$-fold cover of $M_{K}$ corresponding to $\pi_{1}\left(M_{K}\right) \stackrel{\epsilon}{\rightarrow} \mathbb{Z} \rightarrow \mathbb{Z} / k$, and denote by $L_{k}$ the $k$-fold cover of $S^{3}$ branched along $K \subset S^{3}$. Note that $H_{1}\left(L_{k}\right)$ and $H_{1}\left(M_{k}\right)$ also have $\Lambda$-module structures.

\section{Lemma 2.2}

$$
\left|H_{1}\left(L_{k}\right)\right|=\left|\prod_{j=1}^{k} \Delta_{K}\left(e^{2 \pi i j / k}\right)\right|,
$$

where 0 on the right hand side means that $H_{1}\left(L_{k}\right)$ is infinite.

(2) There exist natural isomorphisms

$$
\begin{aligned}
H_{1}\left(L_{k}\right) & =H_{1}\left(M_{K}, \Lambda\right) /\left(t^{k}-1\right), \\
H_{1}\left(M_{k}\right) & =H_{1}\left(L_{k}\right) \oplus \mathbb{Z}=H_{1}\left(M_{K}, \Lambda\right) /\left(t^{k}-1\right) \oplus \mathbb{Z} .
\end{aligned}
$$

Proof The first part is shown in Gor77, p. 17]. For the second part consider the long exact sequence

$$
\cdots \rightarrow H_{i}\left(\tilde{M}_{K}\right) \stackrel{t^{k}-1}{\longrightarrow} H_{i}\left(\tilde{M}_{K}\right) \rightarrow H_{i}\left(M_{k}\right) \rightarrow \ldots
$$

It follows immediately that $H_{1}\left(M_{k}\right)=H_{1}\left(M_{K}, \Lambda\right) /\left(t^{k}-1\right) \oplus \mathbb{Z}$. The other isomorphism is clear.

Now let $k$ be any integer such that $H_{1}\left(L_{k}\right)$ is finite, then the map

$$
\begin{aligned}
\lambda_{L, k}=\lambda_{L}: H_{1}\left(L_{k}\right) \times H_{1}\left(L_{k}\right) & \rightarrow \mathbb{Q} / \mathbb{Z} \\
(a, b) & \mapsto \frac{1}{n} a \cdot c \bmod \mathbb{Z},
\end{aligned}
$$

where $c \in C_{2}\left(L_{k}\right)$ such that $\partial(c)=n b$, defines a symmetric, non-singular pairing which is called the linking pairing of $H_{1}\left(L_{k}\right)=T H_{1}\left(M_{k}\right)$. For any $\mathbb{Z}$-submodule $P_{k} \subset T H_{1}\left(M_{k}\right)$ define

$$
P_{k}^{\perp}:=\left\{v \in T H_{1}\left(M_{k}\right) \mid \lambda_{L}(v, w)=0 \text { for all } w \in P_{k}\right\} .
$$

If $P_{k}$ is a $\Lambda$-submodule with $P_{k}=P_{k}^{\perp}$ then we say that $P_{k}$ is a metabolizer for the linking pairing $\lambda_{L}$. If $\lambda_{L}$ has a metabolizer then we say that $\lambda_{L}$ is metabolic. Note that if $P_{k}$ is a metabolizer for the linking pairing, then $\left|P_{k}\right|^{2}=$ $\left|H_{1}\left(L_{k}\right)\right|$. 


\subsection{Homology of prime-power covers and the linking pairing}

The following corollary shows that prime power covers of manifolds behave in a more 'controlled' way.

Lemma 2.3 Let $Y$ be a manifold such that $H_{*}(Y)=H_{*}\left(S^{1}\right)$ and let $k=p^{s}$ where $p$ is a prime number. Write $Y_{k}$ for the $k$-fold cover of $Y$ corresponding to $H_{1}(Y)=H_{1}\left(S^{1}\right)=\mathbb{Z} \rightarrow \mathbb{Z} / k$. Then $H_{*}\left(Y_{k}\right)=H_{*}(Y) \oplus$ torsion.

The following proof is modelled after CG86, p. 184].

Proof For any $n \in \mathbb{N} \cup\{\infty\}$ we can give $H_{*}\left(Y_{n}\right)$ a $\Lambda$-structure. Since $H_{2}(Y, \mathbb{Z} / p)=0$ we get the following exact sequence

$$
0 \rightarrow H_{1}\left(Y_{\infty}, \mathbb{Z} / p\right) \stackrel{t-1}{\longrightarrow} H_{1}\left(Y_{\infty}, \mathbb{Z} / p\right) \rightarrow H_{1}(Y, \mathbb{Z} / p) \rightarrow H_{0}\left(Y_{\infty}, \mathbb{Z} / p\right) \rightarrow 0 .
$$

Since $H_{1}(Y, \mathbb{Z} / p) \rightarrow H_{0}\left(Y_{\infty}, \mathbb{Z} / p\right)$ is an isomorphism it follows from the sequence that the map $H_{1}\left(Y_{\infty}, \mathbb{Z} / p\right) \stackrel{t-1}{\longrightarrow} H_{1}\left(Y_{\infty}, \mathbb{Z} / p\right)$ is an isomorphism. Since $H_{i}(Y)=0$ for $i>1$ we also get that $H_{i}\left(Y_{\infty}, \mathbb{Z} / p\right) \stackrel{t-1}{\longrightarrow} H_{i}\left(Y_{\infty}, \mathbb{Z} / p\right)$ is an isomorphism for $i>1$. Over $\mathbb{Z} / p$ we get $\left(t^{k}-1\right)=\left(t^{p^{s}}-1\right)=(t-1)^{p^{s}}$, hence multiplication by $\left(t^{k}-1\right)$ is an automorphism of $H_{1}\left(Y_{\infty}, \mathbb{Z} / p\right)$ as well. Consider the long exact sequence

$$
\cdots \rightarrow H_{1}\left(Y_{\infty}, \mathbb{Z} / p\right) \stackrel{t^{k}-1}{\longrightarrow} H_{1}\left(Y_{\infty}, \mathbb{Z} / p\right) \rightarrow H_{1}\left(Y_{k}, \mathbb{Z} / p\right) \rightarrow H_{0}\left(Y_{\infty}, \mathbb{Z} / p\right) \rightarrow 0 .
$$

It follows that $H_{1}\left(Y_{k}, \mathbb{Z} / p\right)=H_{0}\left(Y_{\infty}, \mathbb{Z} / p\right)=\mathbb{Z} / p$. Similarly we can show that $H_{i}\left(Y_{k}, \mathbb{Z} / p\right)=0$ for $i>1$. The lemma now follows from the universal coefficient theorem.

Corollary 2.4 Let $k$ be a prime power.

$$
\begin{aligned}
H_{1}\left(M_{k}\right) & =\mathbb{Z} \oplus T H_{1}\left(M_{k}\right) . \\
T H_{1}\left(M_{k}\right) & =H_{1}\left(L_{k}\right)=H_{1}\left(M_{K}, \Lambda\right) /\left(t^{k}-1\right) .
\end{aligned}
$$

(2) Let $D$ be a slice disk, denote the $k$-fold cover of $N_{D}$ by $N_{k}$, then $H_{1}\left(N_{k}\right)=\mathbb{Z} \oplus T H_{1}\left(N_{k}\right)=\mathbb{Z} \oplus H_{1}(N, \Lambda) /\left(t^{k}-1\right)$.

(3) $Q_{k}:=\operatorname{Ker}\left\{T H_{1}\left(M_{k}\right) \rightarrow T H_{1}\left(N_{k}\right)\right\}$ is a metabolizer for $\lambda_{L}$.

The first two statements are immediate corollaries. The third statement is wellknown (cf. Gor77] or [Fr03d]). It is important though that $k$ is a prime power, since it is crucial in the proof that $H_{1}\left(N_{k}\right)=\mathbb{Z} \oplus T H_{1}\left(N_{k}\right)$. 
Remark Let $K$ be a slice knot, $D$ a slice disk. Then $P:=\operatorname{Ker}\left\{H_{1}\left(M_{K}, \Lambda\right)\right.$ $\left.\rightarrow F H_{1}\left(N_{D}, \Lambda\right)\right\}$ is a metabolizer for the Blanchfield pairing and $Q_{k}:=$ $\operatorname{Ker}\left\{T H_{1}\left(M_{k}\right) \rightarrow T H_{1}\left(N_{k}\right)\right\}$ is a metabolizer for $\lambda_{L, k}$. Furthermore $P_{k}:=$ $\pi(P) \subset H_{1}\left(M_{K}, \Lambda\right) /\left(t^{k}-1\right)$ can be shown to be a metabolizer for $\lambda_{L, k}$. It is an open problem whether $P_{k}=Q_{k}$. If yes, then this would show that all the metabolizers $Q_{k}$ can be lifted to a metabolizer for the Blanchfield pairing, which would resolve the problems which appeared in Gi93 and Let00] (cf. sections 8.3 and 8.4 .

\section{Introduction to eta-invariants and first application to knots}

\subsection{Eta invariants}

Let $C$ be a complex, hermitian matrix, i.e. $C=\bar{C}^{t}$, then the signature $\operatorname{sign}(C)$ is defined as the number of positive eigenvalues of $C$ minus the number of negative eigenvalues. The following is an easy exercise.

Lemma 3.1 If $C$ is hermitian then $\operatorname{sign}\left(P C \bar{P}^{t}\right)=\operatorname{sign}(C)$ for any $P$ with $\operatorname{det}(P) \neq 0$. If furthermore $\operatorname{det}(C) \neq 0$ and $C$ is of the form

$$
C=\left(\begin{array}{cc}
0 & B \\
\bar{B}^{t} & D
\end{array}\right)
$$

where $B$ is a square matrix, then $\operatorname{sign}(C)=0$.

Let $M^{3}$ be a closed manifold and $\alpha: \pi_{1}(M) \rightarrow U(k)$ a representation. Atiyah, Patodi, Singer APS75] associated to $(M, \alpha)$ a number $\eta(M, \alpha)$ called the (reduced) eta invariant of $(M, \alpha)$.

The main theorem to compute the eta invariant is the following.

Theorem 3.2 (Atiyah-Patodi-Singer index theorem [APS75]) If there exists a manifold $W^{4}$ and a representation $\left.\beta: \pi_{1}(W) \rightarrow U(k)\right)$ such that $\partial(W, \beta)=$ $r\left(M^{3}, \alpha\right)$ for some $r \in \mathbb{N}$, then

$$
\eta(M, \alpha)=\frac{1}{r}\left(\operatorname{sign}_{\beta}(W)-k \operatorname{sign}(W)\right),
$$

where $\operatorname{sign}_{\beta}(W)$ denotes the signature of the twisted intersection pairing on $H_{2}^{\beta}(W)$. 


\subsection{Application of eta invariants to knots}

For a knot $K$ we will study the eta invariants associated to the closed manifold $M_{K}$. In the context of knot theory they were studied by Levine Lev94] who used them to find links which are not concordant to boundary links. Letsche Let00 used eta invariants to study knot concordance in dimension three.

Definition For a group $G$ the derived series is defined by $G^{(0)}:=G$ and inductively $G^{(i+1)}:=\left[G^{(i)}, G^{(i)}\right]$ for $i>0$.

The inclusion map $S^{3} \backslash K \rightarrow M_{K}$ defines a homomorphism $\pi_{1}\left(S^{3} \backslash K\right) \rightarrow$ $\pi_{1}\left(M_{K}\right)$, the kernel is generated by the longitude of $K$ which lies in $\pi_{1}\left(S^{3} \backslash\right.$ $K)^{(2)}$. In particular $\pi_{1}\left(S^{3} \backslash K\right) / \pi_{1}\left(S^{3} \backslash K\right)^{(2)} \rightarrow \pi_{1}\left(M_{K}\right) / \pi_{1}\left(M_{K}\right)^{(2)}$ is an isomorphism.

Let $M_{O}$ be the zero-framed surgery on the trivial knot. Then $M_{O}=S^{1} \times S^{2}$ which bounds $S^{1} \times D^{3}$, which is homotopic to a 1-complex. This proves that the unknot has vanishing eta invariant for any unitary representation since any representation of $M_{O}$ extends over $S^{1} \times D^{3}$.

\section{$3.3 \quad U(1)$-representations}

Let $K$ be a knot, $A$ a Seifert matrix, then we define the signature function $\sigma(K): S^{1} \rightarrow \mathbb{Z}$ of $K$ as follows (cf. [Lev69, p. 242])

$$
\sigma_{z}(K):=\operatorname{sign}\left(A(1-z)+A^{t}(1-\bar{z})\right) .
$$

It is easy to see that this is independent of the choice of $A$.

Proposition 3.3 Let $K$ be a knot, $\mu$ a meridian and let $\alpha: \pi_{1}\left(M_{K}\right) \rightarrow U(1)$ be a representation.

(1) Let $z:=\alpha(\mu)$, then

$$
\eta\left(M_{K}, \alpha\right)=\sigma_{z}(K) .
$$

(2) The function $z \mapsto \sigma_{z}(K)$ is locally constant outside of the zero set of $\Delta_{K}(t)$.

(3) If $K$ is algebraically slice and $z \in S^{1}$ such that $\Delta_{K}(z) \neq 0$, then $\sigma_{z}(K)=$ 0 . In particular if $z$ is a prime power root of unity, then $\sigma_{z}(K)=0$.

(4) Given $z$ a prime power root of unity, $K \mapsto \sigma_{z}(K)$ defines a homomorphism from the knot concordance group to $\mathbb{Z}$. 
Proof (1) See [Lit84].

(2) The function $z \mapsto \sigma_{z}(K)$ is continuous on

$$
\left\{z \in S^{1} \mid A(1-z)+A^{t}(1-\bar{z}) \text { is non-singular }\right\} .
$$

an easy argument shows that this set equals

$$
\{1\} \cup\left\{z \in S^{1} \mid \Delta_{K}(z)=\operatorname{det}\left(A z-A^{t}\right) \neq 0\right\} .
$$

Levine [Lev69] showed that the signature function is continuous at $z=1$.

(3) If $z \in S^{1}$ such that $\Delta_{K}(z) \neq 0$ then $A(1-z)+A^{t}(1-\bar{z})$ is non-singular and the first part follows from lemma 3.1. The second part follows from $\Delta_{K}(1)=1$ and the well-known fact that $\Phi_{p^{r}}(1)=p$ for a prime $p$, where $\Phi_{p^{r}}(t)$ denotes the minimal polynomial of a primitive root of unity of order $p^{r}$.

(4) Follows immediately from (3) and the additivity of the twisted signature function.

Let $K$ be a knot, $m \in \mathbb{N}$, then we can form $m K$ by iterated connected sum. We say $K$ is (algebraically) torsion if $m K$ is (algebraically) slice for some $m$. It follows from proposition 3.3 (4) that $\sigma_{z}(K)=0$ for any algebraically torsion knot $K$ and $z$ a prime power root of unity.

Levine [Lev69] showed that for $n>1$ a knot $K \subset S^{2 n+1}$ is slice if and only if $K$ is algebraically slice. Levine Lev69b and Matumuto Ma77 showed that $K$ is algebraically torsion if and only if $\eta\left(M_{K}, \alpha\right)=0$ for all $\alpha: \pi_{1}\left(M_{K}\right) \rightarrow U(1)$ of prime power order. In particular, in the case $n>1$ the $U(1)$-eta invariant detects any non-torsion knot.

In the classical case $n=1$ Casson and Gordon CG86 found an example of a non-slice knot which is algebraically slice. Jian J81 showed using the CassonGordon invariant that there are knots which are algebraically slice and which are not torsion in the knot concordance group.

The goal of this paper is to study to which degree certain non-abelian eta invariants can detect algebraically slice knots which are not topologically slice.

\section{Metabelian eta invariants and the main sliceness obstruction theorem}

A group $G$ is called metabelian if $G^{(2)}=\{e\}$, a representation $\varphi: \pi_{1}(M) \rightarrow$ $U(k)$ is called metabelian if it factors through $\pi_{1}(M) / \pi_{1}(M)^{(2)}$. Metabelian eta 
invariants in the context of knot concordance were studied by Letsche [Let00]. Our approach is influenced by his work.

For a knot $K$ let $\pi:=\pi_{1}\left(M_{K}\right)$, then consider

$$
1 \rightarrow \pi^{(1)} / \pi^{(2)} \rightarrow \pi / \pi^{(2)} \rightarrow \pi / \pi^{(1)} \rightarrow 1 .
$$

Note that $\pi_{1}\left(\tilde{M}_{K}\right)=\pi_{1}\left(M_{K}\right)^{(1)}$ where $\tilde{M}_{K}$ denotes the infinite cyclic cover of $M_{K}$, hence $H_{1}\left(M_{K}, \Lambda\right)=H_{1}\left(\tilde{M}_{K}\right) \cong \pi_{1}\left(M_{K}\right)^{(1)} / \pi_{1}\left(M_{K}\right)^{(2)}$. Since $\pi / \pi^{(1)}=$ $H_{1}\left(M_{K}\right)=\mathbb{Z}$ the above sequence splits and we get isomorphisms

$$
\pi / \pi^{(2)} \cong \pi / \pi^{(1)} \ltimes \pi^{(1)} / \pi^{(2)} \cong \mathbb{Z} \ltimes H_{1}\left(M_{K}, \Lambda\right),
$$

where $n \in \mathbb{Z}$ acts by multiplication by $t^{n}$. This shows that metabelian representations of $\pi_{1}\left(M_{K}\right)$ correspond to representations of $\mathbb{Z} \ltimes H_{1}\left(M_{K}, \Lambda\right)$.

\subsection{Metabelian representations of $\pi_{1}\left(M_{K}\right)$}

For a group $G$ denote by $R_{k}^{i r r}(G)$ (resp. $R_{k}^{\text {irr,met }}(G)$ ) the set of conjugacy classes of irreducible, $k$-dimensional, unitary (metabelian) representations of $G$. Note that the eta invariant of a manifold only depends on the conjugacy class of a unitary representation. Recall that for a knot $K$ we can identify

$$
R_{k}^{i r r, m e t}\left(\pi_{1}\left(M_{K}\right)\right)=R_{k}^{i r r}\left(\mathbb{Z} \ltimes H_{1}\left(M_{K}, \Lambda\right)\right) .
$$

The following proposition gives a very useful classification of all irreducible metabelian unitary representations of $\pi_{1}\left(M_{K}\right)$ (cf. [Let95] for a different approach).

Proposition 4.1 Let $H$ be a $\Lambda$-module. Let $z \in S^{1}$ and $\chi: H \rightarrow H /\left(t^{k}-\right.$ 1) $\rightarrow S^{1}$ a character, then

$$
\begin{aligned}
\alpha_{(z, \chi)}: \mathbb{Z} \ltimes H & \rightarrow U(k) \\
(n, h) & \mapsto z^{n}\left(\begin{array}{cccc}
0 & \ldots & 0 & 1 \\
1 & \ldots & 0 & 0 \\
\vdots & \ddots & & \vdots \\
0 & \ldots & 1 & 0
\end{array}\right)^{n}\left(\begin{array}{cccc}
\chi(h) & 0 & \ldots & 0 \\
0 & \chi(t h) & \ldots & 0 \\
\vdots & & \ddots & \vdots \\
0 & 0 & \ldots & \chi\left(t^{k-1} h\right)
\end{array}\right)
\end{aligned}
$$

defines a representation. If $\chi$ does not factor through $H /\left(t^{l}-1\right)$ for some $l<k$, then $\alpha_{(z, \chi)}$ is irreducible.

Conversely, any $[\alpha] \in R_{k}^{\text {irr }}(\mathbb{Z} \ltimes H)$ has a representative $\alpha_{(z, \chi)}$ with $z, \chi$ as above. 
Proof It is easy to check that $\alpha_{(z, \chi)}$ is well-defined. Now let $[\alpha] \in R_{k}^{i r r}(\mathbb{Z} \ltimes H)$. Denote by $\chi_{1}, \ldots, \chi_{l}: H \rightarrow S^{1}$ the different weights of $\alpha: 0 \times H \rightarrow U(k)$. Since $H$ is an abelian group we can write $\mathbb{C}^{k}=\oplus_{i=1}^{l} V_{\chi_{i}}$ where $V_{\chi_{i}}:=\{v \in$ $\mathbb{C}^{k} \mid \alpha(0, h)(v)=\chi_{i}(h) v$ for all $\left.h\right\}$ is the weight space corresponding to $\chi_{i}$.

Recall that the group structure of $\mathbb{Z} \ltimes H$ is given by

$$
(n, h)(m, k)=\left(n+m, t^{m} h+k\right) .
$$

In particular for all $v \in H$

$$
(j, 0)\left(0, t^{j} h\right)=\left(j, t^{j} h\right)=(0, h)(j, 0),
$$

therefore for $A:=\alpha(1,0)$ we get

$$
A^{j} \alpha\left(0, t^{j} h\right)=\alpha(j, 0) \alpha\left(0, t^{j} h\right)=\alpha\left(j, t^{j} h\right)=\alpha(0, h) \alpha(j, 0)=\alpha(0, h) A^{j} .
$$

This shows that $\alpha\left(0, t^{j} h\right)=A^{-j} \alpha(0, h) A^{j}$. Now let $v \in V_{\chi(h)}$, then

$$
\alpha(0, h) A v=A \alpha(0, t h) A^{-1} A v=A \alpha(0, t h) v=A \chi(t h) v=\chi(t h) A v,
$$

i.e. $\alpha(1,0): V_{\chi_{i}(h)} \rightarrow V_{\chi_{i}(t h)}$. Since $\alpha$ is irreducible it follows that, after reordering, $\chi_{i}(v)=\chi_{1}\left(t^{i} v\right)$ for all $i=1, \ldots, l$. Note that $A^{j}$ induces isomorphisms between the weight spaces $V_{\chi_{i}}$ and that $A^{l}: V_{\chi_{1}} \rightarrow V_{\chi_{1}}$ is a unitary transformation. In particular it has an eigenvector $v$, hence $\mathbb{C} v \oplus \mathbb{C} A v \oplus \ldots \mathbb{C} A^{l-1} v$ spans an $\alpha$-invariant subspace. Since $\alpha$ is irreducible it follows that $l=k$ and that each $V_{\chi_{i}}$ is one-dimensional.

Since $\alpha$ is a unitary representation we can find a unitary matrix $P$ such that $P \mathbb{C} e_{i}=V_{i}$, in particular, $\alpha_{1}:=P^{-1} \alpha P$ has the following properties.

(1) $\alpha(0 \times H)=\operatorname{diag}\left(\chi(h), \chi(t h), \ldots, \chi\left(t^{k-1} h\right)\right)$,

(2) for some $z_{1}, \ldots, z_{k} \in S^{1}$

$$
\alpha(1,0):=\left(\begin{array}{cccc}
0 & \ldots & 0 & z_{k} \\
z_{1} & \ldots & 0 & 0 \\
\vdots & \ddots & & \vdots \\
0 & \ldots & z_{k-1} & 0
\end{array}\right)
$$

Here we denote by $\operatorname{diag}\left(b_{1}, \ldots, b_{k}\right)$ the diagonal matrix with entries $b_{1}, \ldots, b_{k}$.

Let $z:=\prod_{i=1}^{k} z_{i}$ and let $Q:=\operatorname{diag}\left(d_{1}, \ldots, d_{k}\right)$ where $d_{i}:=\frac{\prod_{j=1}^{i-1} z_{j}}{z^{i-1}}$. Then $\alpha_{2}:=Q^{-1} \alpha_{1} Q$ has the required properties. 


\subsection{Eta invariants as concordance invariants}

We quote some definitions, initially introduced by Levine [Lev94]. Let $G$ be a group, then a $G$-manifold is a pair $(M, \alpha)$ where $M$ is a compact oriented manifold and $\alpha$ is a homomorphism $\alpha: \pi_{1}(M) \rightarrow G$ defined up to inner automorphism.

We call two $G$-manifolds $\left(M_{j}, \alpha_{j}\right), j=1,2$, homology $G$-bordant if there exists a $G$-manifold $\left(N, \beta: \pi_{1}(N) \rightarrow G\right)$ such that $\partial(N)=M_{1} \cup-M_{2}, H_{*}\left(N, M_{j}\right)=0$ for $j=1,2$ and, up to inner automorphisms of $G, \beta \mid \pi_{1}\left(M_{j}\right)=\alpha_{j}$.

We will compare eta invariants for homology $G$-bordant $G$-manifolds.

Definition For a $\Lambda$-torsion module $H$ define $P_{k}^{i r r}(\mathbb{Z} \ltimes H)$ to be the set of conjugacy classes of representations which are conjugate to $\alpha_{(z, \chi)}$ with $z \in S^{1}$ transcendental and $\chi: H /\left(t^{k}-1\right) \rightarrow S^{1}$ factoring through a group of prime power order. If $W$ is a manifold with $H_{1}(W) \cong \mathbb{Z}$ then we define $P_{k}^{i r r, m e t}\left(\pi_{1}(W)\right):=$ $P_{k}^{i r r}\left(\mathbb{Z} \ltimes H_{1}(W, \Lambda)\right)$.

We need the following theorem, which is a slight reformulation of a theorem by Letsche [Let00] which in turn is based on work by Levine Lev94.

Theorem 4.2 Let $H$ be a $\Lambda$-torsion module, $G:=\mathbb{Z} \ltimes H$. If $\left(M_{1}, \alpha_{1}\right),\left(M_{2}, \alpha_{2}\right)$ are homology $G$-bordant 3-manifolds and if $\theta \in P_{k}^{i r r}(G)$, then $\eta\left(M_{1}, \theta \circ \alpha_{1}\right)=$ $\eta\left(M_{2}, \theta \circ \alpha_{2}\right)$.

Proof Write $\mathcal{P}_{k}\left(\pi_{1}(M)\right)$ for the set of unitary representations $\alpha: \pi_{1}(M) \rightarrow$ $U(k)$ with the following two properties (cf. [Let00, p. 311])

(1) $\alpha$ factors through a non-abelian group of the form $\mathbb{Z} \ltimes P, P$ a finite $p$-group and $\mathbb{Z} \ltimes P \rightarrow \mathbb{Z}$ induces an isomorphism on first homology,

(2) there exists $g \in \pi_{1}(M)$ that generates $\pi_{1}(M)$ such that all eigenvalues of $\alpha(g)$ are transcendental.

Letsche [Let00, prop. 1.7, cor. 3.10, thm. 3.11] showed that the statement holds for all $\theta \in \mathcal{P}_{k}(G)$. Clearly if $\alpha \in \mathcal{P}_{k}(G)$, then all its conjugates lie in $\mathcal{P}_{k}(G)$ as well.

It therefore suffices to show that $\alpha_{(z, \chi)} \in \mathcal{P}_{k}(\mathbb{Z} \ltimes H)$ if $\left[\alpha_{(z, \chi)}\right] \in P_{k}^{\text {irr }}(\mathbb{Z} \ltimes H)$. Let $\left[\alpha_{(z, \chi)}\right] \in P_{k}^{i r r}(\mathbb{Z} \ltimes H)$, i.e. $z \in S^{1}$ transcendental and $\chi: H /\left(t^{k}-1\right) \rightarrow$ $\mathbb{Z} / m \rightarrow S^{1}$ where $m$ is a prime power. Then the result follows immediately from the observations that 
(1) all the eigenvalues of $\alpha_{(z, \chi)}(1,0)$ are of the form $z e^{2 \pi i j / k}$, in particular all are transcendental,

(2) $\quad \alpha_{(z, \chi)}: \mathbb{Z} \ltimes H \rightarrow U(k)$ factors through $\mathbb{Z} \ltimes(\mathbb{Z} / m)^{k}$ and $(\mathbb{Z} / m)^{k}$ is a group of prime power order, where $\mathbb{Z}$ acts on $(\mathbb{Z} / m)^{k}$ by cyclic permutation, i.e. by $1 \cdot\left(v_{1}, \ldots, v_{k}\right):=\left(v_{k}, v_{1}, \ldots, v_{k-1}\right)$,

(3) $\quad H_{1}\left(\mathbb{Z} \ltimes(\mathbb{Z} / m)^{k}\right) \rightarrow H_{1}(\mathbb{Z})$ is an isomorphism.

Let $K$ be a slice knot with slice disk $D$ and let $\alpha_{(z, \chi)} \in R_{k}^{i r r, m e t}\left(\pi_{1}\left(M_{K}\right)\right)$. Consider the following diagram

$$
\begin{aligned}
& \pi_{1}\left(M_{K}\right) \rightarrow \mathbb{Z} \ltimes H_{1}\left(M_{K}, \Lambda\right) \rightarrow \mathbb{Z} \ltimes H_{1}\left(M_{K}, \Lambda\right) /\left(t^{k}-1\right) \stackrel{\alpha_{(z, \chi)}}{\longrightarrow} U(k)
\end{aligned}
$$

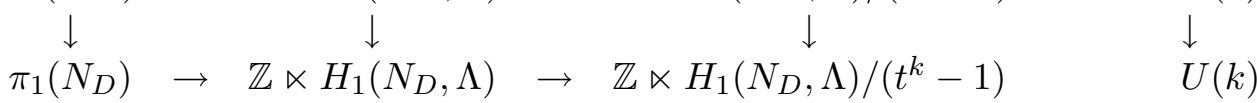

If $\chi$ vanishes on $\operatorname{Ker}\left\{H_{1}\left(M_{K}, \Lambda\right) /\left(t^{k}-1\right) \rightarrow H_{1}\left(N_{D}, \Lambda\right) /\left(t^{k}-1\right)\right\}$ then $\chi$ extends to $\chi_{N}: H_{1}\left(N_{D}, \Lambda\right) /\left(t^{k}-1\right) \rightarrow S^{1}$ since $S^{1}$ is divisible. Furthermore, if $\chi$ is of prime power order, then $\chi_{N}$ can be chosen to be of prime power order as well. Note that $\alpha_{\left(z, \chi_{N}\right)}: \pi_{1}\left(N_{D}\right) \rightarrow U(k)$ is an extension of $\alpha_{(z, \chi)}: \pi_{1}\left(M_{K}\right) \rightarrow U(k)$. This proves the following.

Lemma 4.3 (1) $\alpha_{(z, \chi)}$ extends to a metabelian representation of $\pi_{1}\left(N_{D}\right)$ if and only if $\chi$ vanishes on $\operatorname{Ker}\left\{H_{1}\left(M_{K}, \Lambda\right) /\left(t^{k}-1\right) \rightarrow H_{1}\left(N_{D}, \Lambda\right) /\left(t^{k}-\right.\right.$ $1)\}$.

(2) If $\alpha_{(z, \chi)} \in P_{k}^{i r r}\left(\pi_{1}\left(M_{K}\right)\right)$ extends to a metabelian representation of $\pi_{1}\left(N_{D}\right)$ then $\alpha_{(z, \chi)}$ extends to a representation in $P_{k}^{i r r}\left(\pi_{1}\left(N_{D}\right)\right)$.

Theorem 4.4 Let $K$ be a slice knot and $D$ a slice disk. If $\alpha$ extends to a metabelian representation of $\pi_{1}\left(N_{D}\right)$ and if $\alpha \in P_{k}^{i r r}\left(\pi_{1}\left(M_{K}\right)\right)$, then $\eta\left(M_{K}, \alpha\right)=0$.

Proof By lemma 4.3 we can find an extension $\beta \in P_{k}^{i r r}\left(\pi_{1}\left(N_{D}\right)\right)$. We can decompose $N_{D}$ as $N_{D}=W^{4} \cup_{M_{O}} S^{1} \times D^{3}$ where $M_{O}=S^{1} \times S^{2}$ is the zeroframed surgery along the trivial knot in $S^{3}$ and $W$ is a homology $\mathbb{Z}$-bordism between $M_{K}$ and $M_{O}$. The statement now follows from theorem 4.2 and the fact that the unknot has zero eta invariants, since $(W$,id) is a homology $\mathbb{Z} \ltimes$ $H_{1}(W, \Lambda)$-bordism between $\left(M_{K}, i_{*}\right)$ and $\left(M_{O}, i_{*}\right)$. 


\subsection{Main sliceness obstruction theorem}

Theorem 4.5 Let $K$ be a slice knot, $k$ a prime power. Then there exists a metabolizer $P_{k} \subset T H_{1}\left(M_{k}\right)$ for the linking pairing, such that for any representation $\alpha: \pi_{1}\left(M_{K}\right) \rightarrow \mathbb{Z} \ltimes H_{1}\left(M_{K}, \Lambda\right) /\left(t^{k}-1\right) \rightarrow U(k)$ vanishing on $0 \times P_{k}$ and lying in $P_{k}^{\text {irr,met }}\left(\pi_{1}\left(M_{K}\right)\right)$ we get $\eta\left(M_{K}, \alpha\right)=0$.

Proof Let $D$ be a slice disk. Let

$$
P_{k}:=\operatorname{Ker}\left\{H_{1}\left(M_{K}, \Lambda\right) /\left(t^{k}-1\right) \rightarrow H_{1}\left(N_{D}, \Lambda\right) /\left(t^{k}-1\right)\right\},
$$

this is a metabolizer for the linking pairing by proposition 2.4. The theorem now follows from lemma 4.3 and theorem 4.4

In the following we will show that some eta-invariants of slice knots vanish for non-prime power dimensional irreducible representations. Let $\alpha_{1} \in$ $R_{k_{1}}(G), \alpha_{2} \in R_{k_{2}}(G)$, then we can form the tensor product $\alpha_{1} \otimes \alpha_{2} \in R_{k_{1} k_{2}}(G)$.

Proposition 4.6 If $k_{1}, k_{2}$ are coprime, then

$$
\alpha_{\left(k_{1}, z_{1}, \chi_{1}\right)} \otimes \alpha_{\left(k_{2}, z_{2}, \chi_{2}\right)} \cong \alpha_{\left(k_{1} k_{2}, z_{1} z_{2}, \chi_{1} \chi_{2}\right)} .
$$

If furthermore $\alpha_{\left(k_{1}, z_{1}, \chi_{1}\right)}, \alpha_{\left(k_{2}, z_{2}, \chi_{2}\right)}$ are irreducible, then $\alpha_{\left(k_{1}, z_{1}, \chi_{1}\right)} \otimes \alpha_{\left(k_{2}, z_{2}, \chi_{2}\right)}$ is irreducible as well.

Proof Denote by $e_{11}, \ldots, e_{k_{1} 1}$ and $e_{12}, \ldots, e_{k_{2} 2}$ the canonical bases of $\mathbb{C}^{k_{1}}$ and $\mathbb{C}^{k_{2}}$. Set $f_{i}:=e_{i \bmod k_{1}, 1} \otimes e_{i \bmod k_{2}, 2}$ for $i=0, \ldots, k_{1} k_{2}-1$. The $f_{i}$ 's are distinct, therefore $\left\{f_{i}\right\}_{i=0, \ldots, k_{1} k_{2}-1}$ form a basis for $\mathbb{C}^{k_{1}} \otimes \mathbb{C}^{k_{2}}$. One can easily see that $\alpha_{1} \otimes \alpha_{2}$ with respect to this basis is just $\alpha_{\left(k_{1} k_{2}, z_{1} z_{2}, \chi_{1} \chi_{2}\right)}$.

The last statement follows from the observation that if $\chi_{1} \chi_{2}: H /\left(t^{k_{1} k_{2}}-1\right)$ factors through $H /\left(t^{k}-1\right)$ for some $k<k_{1} k_{2}$, then one of the $\chi_{i}: H \rightarrow$ $H /\left(t^{k_{i}}-1\right)$ factors through $H /\left(t^{k}-1\right)$ for some $k<k_{i}$.

For a prime number $p$ and a $\Lambda$-module $H$ denote by $P_{k, p}^{i r r}(\mathbb{Z} \ltimes H)$ the set of representations $\alpha_{(z, \chi)}$ in $P_{k}^{i r r}(\mathbb{Z} \ltimes H)$ where $\chi$ factors through a $p$-group. Define $P_{k, p}^{i r r, m e t}\left(\pi_{1}\left(M_{K}\right)\right):=P_{k, p}^{i r r}\left(\mathbb{Z} \ltimes H_{1}\left(M_{K}, \Lambda\right)\right)$.

Theorem 4.7 Let $K$ be a slice knot, $k_{1}, \ldots, k_{r}$ pairwise coprime prime powers, then there exist metabolizers $P_{k_{i}} \subset T H_{1}\left(M_{k_{i}}\right), i=1, \ldots, r$ for the linking pairings, such that for any prime number $p$ and any choice of irreducible representations $\alpha_{i}: \pi_{1}\left(M_{K}\right) \rightarrow \mathbb{Z} \ltimes H_{1}\left(M_{K}, \Lambda\right) /\left(t^{k_{i}}-1\right) \rightarrow U\left(k_{i}\right)$ vanishing on $0 \times P_{k_{i}}$ and lying in $P_{k_{i}, p}^{i r r, m e t}\left(\pi_{1}\left(M_{K}\right)\right)$ we get $\eta\left(M_{K}, \alpha_{1} \otimes \cdots \otimes \alpha_{r}\right)=0$. 
Proof Let $D$ be a slice disk and let $P_{k_{i}}:=\operatorname{Ker}\left\{H_{1}\left(M_{K}, \Lambda\right) /\left(t^{k_{i}}-1\right) \rightarrow\right.$ $\left.H_{1}\left(N_{D}, \Lambda\right) /\left(t^{k_{i}}-1\right)\right\}$. All the representations $\alpha_{1}, \ldots, \alpha_{r}$ extend to metabelian representations of $N_{D}$, hence $\alpha_{1} \otimes \cdots \otimes \alpha_{r}$ also extends to a metabelian representation of $N_{D}$. Write $\alpha=\alpha_{\left(z_{i}, \chi_{i}\right)}$, then $\alpha_{1} \otimes \cdots \otimes \alpha_{r}=\alpha_{\left(z_{1} \cdots z_{r}, \chi_{1} \cdots \chi_{r}\right)}$ since the $k_{i}$ are pairwise coprime. This shows that $\alpha_{1} \otimes \cdots \otimes \alpha_{r} \in P_{k_{1} \cdots \cdot k_{r}, p}^{i r r, m e t}\left(\pi_{1}\left(M_{K}\right)\right)$, therefore $\eta\left(M_{K}, \alpha_{1} \otimes \cdots \otimes \alpha_{r}\right)=0$ by theorem 4.4

Remark Theorem 4.4 holds in fact for locally flat slice disks, and therefore theorem 4.7 holds in fact for topologically slice knots. Indeed, let $D$ be a topological slice disk for $K$, i. e. an embedding $D \subset D^{4}$ such that $\partial(D)=$ $K$ and such that $D$ is locally flat, i.e. there exists an embedding $f: D \times$ $D^{2} \rightarrow D^{4}$ which extends the embedding $D \subset D^{4}$. We write again $N_{D}:=$ $\overline{D^{4} \backslash f\left(D \times D^{2}\right)}$. Let $\alpha \in P_{k}^{i r r, m e t}\left(\pi_{1}\left(M_{K}\right)\right)$ which extends to a representation $\beta \in P_{k}^{\text {irr,met }}\left(\pi_{1}\left(N_{D}\right)\right)$. We can conclude that $\operatorname{sign}_{\beta}\left(N_{D}\right)-k \operatorname{sign}\left(N_{D}\right)=0$. Since $N_{D}$ is in general not a smooth manifold we can not appeal to theorem 3.2 to conclude that $\eta\left(M_{K}, \alpha\right)=0$.

Now consider the Kirby-Siebenmann invariant $k s\left(N_{D}\right)$ of $N_{D}$. By Ra96 p. 10] we have $k s\left(N_{D}\right)=\frac{1}{8} \operatorname{sign}\left(N_{D}\right)-\mu\left(M_{K}\right)$ where $\mu\left(M_{K}\right)$ denotes the Rochlin invariant. Note that $\mu\left(M_{K}\right)$ equals the Arf invariant for $K$, which vanishes for topologically slice knots. Therefore $k s\left(N_{D}\right)=0$, by [FQ90, p. 125] there exists an $r$ such that $N_{D} \# r S^{2} \times S^{2}$ is smooth. Hence we get

$$
\begin{aligned}
\eta\left(M_{K}, \alpha\right) & =\operatorname{sign}_{\beta}\left(N_{D} \# r S^{2} \times S^{2}\right)-k \operatorname{sign}\left(N_{D} \# r S^{2} \times S^{2}\right) \\
& =\operatorname{sign}_{\beta}\left(N_{D}\right)-k \operatorname{sign}\left(N_{D}\right)=0,
\end{aligned}
$$

where the second to last equality follows from Novikov signature additivity, and the observation that

$$
\operatorname{sign}_{\beta}\left(r S^{2} \times S^{2}\right)=k \operatorname{sign}\left(r S^{2} \times S^{2}\right)
$$

since $\beta$ is a trivial $k$-dimensional representation.

Proposition 4.6] shows that $\alpha_{1} \otimes \cdots \otimes \alpha_{r}$ is irreducible since $\operatorname{gcd}\left(k_{1}, \ldots, k_{r}\right)=1$, i.e. the theorem shows that certain non-prime-power dimensional irreducible eta invariants vanish for slice knots. Letsche [Let00 pointed out the fact that non prime power dimensional representations can give sliceness (ribbonness) obstructions. In his thesis [Fr03d] the author shows that in fact Letsche's non prime power dimensional representations are tensor products of prime power dimensional representations.

We say that a knot $K$ has zero slice-eta-obstruction (SE-obstruction) if the conclusion of theorem 4.5 holds for all prime powers $k$, and $K$ has zero slicetensor-eta-obstruction (STE-obstruction) if the conclusion of theorem 4.7 holds for all pairwise coprime prime powers $k_{1}, \ldots, k_{r}$. 
Question 4.8 Are there examples of knots which have zero SE-obstruction but non zero STE-obstruction?

Remark It is easy to find examples of a knot $K$ and one-dimensional representations $\alpha, \beta$ such that $\eta\left(M_{K}, \alpha\right)=0$ and $\eta\left(M_{K}, \beta\right)=0$ but $\eta\left(M_{K}, \alpha \otimes \beta\right) \neq 0$. This shows that in general $\eta\left(M_{K}, \alpha \otimes \beta\right)$ is not determined by $\eta\left(M_{K}, \alpha\right)$ and $\eta\left(M_{K}, \beta\right)$.

Note that if $T H_{1}\left(M_{k}\right)=0$, then $R_{k}^{\text {irr } r \text { met }}\left(\pi_{1}\left(M_{K}\right)\right)=\emptyset$, therefore theorem 4.7 only gives a non-trivial sliceness obstruction if $T H_{1}\left(M_{k}\right) \neq 0$ for some prime power $k$. This is not always the case, in fact Livingston proved the following theorem.

Theorem 4.9 Liv02, thm. 1.2] Let $K$ be a knot. There exists a prime power $k$ with $T H_{1}\left(M_{k}\right) \neq 0$ if and only if $\Delta_{K}(t)$ has a non-trivial irreducible factor that is not an $n$-cyclotomic polynomial with $n$ divisible by three distinct primes.

In section [7.4 we will use this theorem to show that there exists a knot $K$ with $H_{1}\left(L_{k}\right)=0$ for all prime powers $k$, but $H_{1}\left(L_{6}\right) \neq 0$. This shows that $\pi_{1}\left(M_{K}\right)$ has irreducible $U(6)$-representations, but no unitary irreducible representations of prime power dimensions. In particular not all representations are tensor products of prime power dimensional representations.

\section{Casson-Gordon obstruction}

\subsection{The Casson-Gordon obstruction to a knot being slice}

We first recall the definition of the Casson-Gordon obstructions (cf. [CG86]). For $m$ a number denote by $C_{m} \subset S^{1}$ the unique cyclic subgroup of order $m$. For a surjective character $\chi: H_{1}\left(M_{k}\right) \rightarrow H_{1}\left(L_{k}\right) \rightarrow C_{m}$, set $F_{\chi}:=\mathbb{Q}\left(e^{2 \pi i / m}\right)$.

Since $\Omega_{3}\left(\mathbb{Z} \times C_{m}\right)=H_{3}\left(\mathbb{Z} \times C_{m}\right)$ is torsion (cf. [CF64] ) there exists a 4-manifold $V_{k}$ and maps $\epsilon^{\prime}: \pi_{1}\left(V_{k}\right) \rightarrow \mathbb{Z}, \chi^{\prime}: \pi_{1}\left(V_{k}\right) \rightarrow C_{m}$ such that $\partial\left(V_{k}, \epsilon^{\prime} \times \chi^{\prime}\right)=$ $r\left(M_{k}, \epsilon \times \chi\right)$ for some $r \in \mathbb{N}$. To simplify notation we will denote the maps $\epsilon^{\prime}$ and $\chi^{\prime}$ on $\pi_{1}\left(V_{k}\right)$ by $\epsilon$ and $\chi$ as well.

The (surjective) map $\epsilon \times \chi: \pi_{1}\left(V_{k}\right) \rightarrow \mathbb{Z} \times C_{m}$ defines a $\left(\mathbb{Z} \times C_{m}\right)$-cover $\tilde{V}_{\infty}$ of $V$. Then $H_{2}\left(C_{*}\left(\tilde{V}_{\infty}\right)\right)$ and $F_{\chi}(t)$ have a canonical $\mathbb{Z}\left[\mathbb{Z} \times C_{m}\right]$-module structure 
and we can form $H_{2}\left(C_{*}\left(\tilde{V}_{\infty}\right) \otimes_{\mathbb{Z}\left[\mathbb{Z} \times C_{m}\right]} F_{\chi}(t)\right)=: H_{*}\left(V_{k}, F_{\chi}(t)\right)$. Since $F_{\chi}(t)$ is flat over $\mathbb{Z}\left[\mathbb{Z} \times C_{m}\right]$ by Maschke's theorem (cf. [La93]) we get

$$
H_{*}\left(V_{k}, F_{\chi}(t)\right)=H_{*}\left(C_{*}\left(\tilde{V}_{\infty}\right) \otimes_{\mathbb{Z}\left[\mathbb{Z} \times C_{m}\right]} F_{\chi}(t)\right) \cong H_{*}\left(\tilde{V}_{\infty}\right) \otimes_{\mathbb{Z}\left[\mathbb{Z} \times C_{m}\right]} F_{\chi}(t),
$$

which is a free $F_{\chi}(t)$-module. If $\chi$ is a character of prime power order, then the $F_{\chi}(t)$-valued intersection pairing on $H_{2}\left(V_{k}, F_{\chi}(t)\right)$ is non-singular (cf. CG86, p. 190]) and therefore defines an element $t\left(V_{k}\right) \in L_{0}\left(F_{\chi}(t)\right)$, the Witt group of non-singular hermitian forms over $F_{\chi}(t)$ (cf. La93, [Ra98). Denote the image of the ordinary intersection pairing on $H_{2}\left(V_{k}\right)$ under the map $L_{0}(\mathbb{C}) \rightarrow$ $L_{0}\left(F_{\chi}(t)\right)$ by $t_{0}\left(V_{k}\right)$.

Casson and Gordon [CG86] show that if $\chi: H_{1}\left(L_{k}\right) \rightarrow C_{m}$ is a character with $m$ a prime power, then

$$
\tau(K, \chi):=\frac{1}{r}\left(t\left(V_{k}\right)-t_{0}\left(V_{k}\right)\right) \in L_{0}\left(F_{\chi}(t)\right) \otimes_{\mathbb{Z}} \mathbb{Q}
$$

is a well-defined invariant of $\left(M_{k}, \epsilon \times \chi\right)$, i.e. independent of the choice of $V_{k}$. Furthermore they prove the following theorem.

Theorem 5.1 CG86, p. 192] Let $k$ be a prime power. If $K \subset S^{3}$ is slice then there exists a metabolizer $P_{k}$ for the linking pairing, such that for any $\chi: T H_{1}\left(M_{k}\right) \rightarrow C_{m}, m$ a prime power, with $\chi\left(P_{k}\right) \equiv 0$ we get $\tau(K, \chi)=0$.

\subsection{Interpretation of Casson-Gordon invariants as eta invari- ants of $M_{k}$}

For a number field $F \subset \mathbb{C}$ we consider $F$ with complex involution and $F(t)$ with the involution given by complex involution and $\bar{t}=t^{-1}$. For $z \in S^{1}$ transcendental and $\tau \in L_{0}(F(t))$ we can consider $\tau(z) \in L_{0}(\mathbb{C})$. Note that sign : $L_{0}(\mathbb{C}) \rightarrow \mathbb{Z}$ defines an isomorphism.

Let $K$ be a knot, $k$ any number, $m$ a prime power and $\chi: H_{1}\left(L_{k}\right) \rightarrow C_{m}$ a character and $\left(V_{k}^{4}, \epsilon \times \chi: \pi_{1}\left(V_{k}\right) \rightarrow \mathbb{Z} \times C_{m}\right)$ such that $\partial\left(V_{k}, \epsilon \times \chi\right)=r\left(M_{k}, \epsilon \times\right.$ $\chi)$.

For a character $\chi: H_{1}\left(L_{k}\right) \rightarrow C_{m}$ define characters $\chi^{j}$ by setting $\chi^{j}(v):=$ $\chi(v)^{j}$, for $z \in S^{1}$ define

$$
\begin{aligned}
& \beta_{\left(z, \chi^{j}\right)}: \pi_{1}\left(M_{k}\right) \quad \rightarrow \quad H_{1}\left(M_{k}\right)=\mathbb{Z} \oplus H_{1}\left(L_{k}\right) \quad \rightarrow \quad S^{1}=U(1) \\
& (n, v) \quad \mapsto z^{n} \chi^{j}(v) .
\end{aligned}
$$


Proposition 5.2 If $z \in S^{1}$ transcendental, then

$$
\operatorname{sign}(\tau(K, \chi)(z))=\eta\left(M_{K}, \beta_{\left(z, \chi^{1}\right)}\right) .
$$

Proof Let $z \in S^{1}$ transcendental, define $\theta: \mathbb{Z} \times C_{m} \rightarrow S^{1}$ by $\theta(n, y):=z^{n} y$. Then

$$
\partial\left(V_{k}, \theta \circ(\epsilon \times \chi)\right)=r\left(M_{k}, \theta \circ(\epsilon \times \chi)\right)=r\left(M_{k}, \beta_{\left(z, \chi^{1}\right)}\right) .
$$

We view $\mathbb{C}$ as a $\mathbb{Z}\left[\mathbb{Z} \times C_{m}\right]$-module via $\theta \circ(\epsilon \times \chi)$ and $\mathbb{C}$ as an $F_{\chi}(t)$ module via evaluating $t$ to $z$. Note that both modules are flat by Maschke's theorem, hence

$$
\begin{aligned}
H_{2}^{\beta}\left(V_{k}, \mathbb{C}\right) & =H_{2}\left(C_{*}\left(\tilde{V}_{\infty}\right)\right) \otimes_{\mathbb{Z}\left[\mathbb{Z} \times C_{m}\right]} \mathbb{C} \\
& =\left(H_{2}\left(C_{*}\left(\tilde{V}_{\infty}\right)\right) \otimes_{\mathbb{Z}\left[\mathbb{Z} \times C_{m}\right]} F_{\chi}(t)\right) \otimes_{F_{\chi}(t)} \mathbb{C} \\
& =H_{2}\left(V_{k}, F_{\chi}(t)\right) \otimes_{F_{\chi}(t)} \mathbb{C} .
\end{aligned}
$$

This also defines an isometry between the forms, i.e. $\operatorname{sign}_{\beta}\left(V_{k}\right)=\operatorname{sign}\left(V_{k}(z)\right)$. This shows that

$$
\begin{aligned}
r \eta\left(M_{k}, \beta\left(z, \chi^{1}\right)\right) & =\operatorname{sign}_{\beta}\left(V_{k}\right)-\operatorname{sign}\left(V_{k}\right)= \\
& =\operatorname{sign}\left(t\left(V_{k}\right)(z)\right)-\operatorname{sign}\left(t_{0}\left(V_{k}\right)\right)=r \operatorname{sign}(\tau(K, \chi)(z)) .
\end{aligned}
$$

Remark The eta invariant carries potentially more information than the function $z \mapsto \operatorname{sign}(\tau(K, \chi)(z))$, since for non-transcendental $z \in S^{1}$ the number $\tau_{z}(K, \chi)$ is not defined, whereas $\eta\left(M_{k}, \beta_{\left(z, \chi_{1}\right)}\right)$ is still defined. For example the $U(1)$-signatures for slice knots are zero outside the set of singularities, but the eta invariant at the singularities contains information about knots being doubly slice (cf. section 3.3).

Proposition 5.3 Let $K$ be a knot, $k$ any number, $m$ a prime power and $\chi: H_{1}\left(L_{k}\right) \rightarrow C_{m}$ a character, then the following are equivalent.

(1) $\tau(K, \chi)=0 \in L_{0}\left(F_{\chi}(t)\right) \otimes_{\mathbb{Z}} \mathbb{Q}$.

(2) $\operatorname{sign}(\rho(\tau(K, \chi))(z))=0 \in \mathbb{Q}$ for all transcendental $z \in S^{1}, \rho \in \operatorname{Gal}\left(F_{\chi}, \mathbb{Q}\right)$.

(3) $\eta\left(M_{k}, \beta_{\left(z, \chi^{j}\right)}\right)=0 \in \mathbb{Z}$ for all $(j, m)=1$, all transcendental $z \in S^{1}$.

Proof The equivalence of (1) and (2) is a purely algebraic statement, which is shown in a separate paper (cf. [Fr03]) using results of Ranicki's Ra98. The equivalence of (2) and (3) follows from proposition 5.2 and the observation that if $\rho \in \operatorname{Gal}\left(F_{\chi}, \mathbb{Q}\right)$ sends $e^{2 \pi i / m}$ to $e^{2 \pi i j / m}$ for some $(j, m)=1$, then $\rho(\tau(K, \chi))=\tau\left(K, \chi^{j}\right)$ and hence

$$
\operatorname{sign}(\rho(\tau(K, \chi))(z))=\operatorname{sign}\left(\tau\left(K, \chi^{j}\right)(z)\right)=\eta\left(M_{k}, \beta_{\left(z, \chi^{j}\right)}\right) .
$$




\subsection{Interpretation of Casson-Gordon invariants as eta invari- ants of $M_{K}$}

The goal is to prove a version of proposition 5.3 with eta invariants of $M_{K}$ instead of eta invariants of $M_{k}$.

Proposition 5.4 Let $K$ be a knot, $z \in S^{1}$ and $\chi: H_{1}\left(M_{k}\right) \rightarrow H_{1}\left(L_{k}\right) \rightarrow C_{m}$ a character. Let $\beta:=\beta_{(z, \chi)}: \pi_{1}\left(M_{k}\right) \rightarrow U(1)$ and $\alpha=\alpha_{(z, \chi)}: \pi_{1}\left(M_{K}\right) \rightarrow$ $U(k)$, then

$$
\eta\left(M_{K}, \alpha\right)-\eta\left(M_{k}, \beta\right)=\sum_{j=1}^{k} \sigma_{e^{2 \pi i j / k}}(K) .
$$

If $K$ is algebraically slice and $H_{1}\left(L_{k}\right)$ is finite, then $\eta\left(M_{K}, \alpha\right)=\eta\left(M_{k}, \beta\right)$.

Proof In Fr03b we show that if $M_{G} \rightarrow M$ is a $G$-cover and $\alpha_{G}: \pi_{1}\left(M_{G}\right) \rightarrow$ $U(1)$ is a representation then

$$
\eta\left(M_{G}, \alpha_{G}\right)=\eta(M, \alpha)-\eta(M, \alpha(G)),
$$

where $\alpha(G): \pi_{1}(M) \rightarrow G \rightarrow U(\mathbb{C}[G])=U\left(\mathbb{C}^{|G|}\right)$ is given by left multiplication and $\alpha: \pi_{1}(M) \rightarrow U(k)$ is the (induced) representation given by

$$
\begin{aligned}
\alpha: \pi_{1}(M) & \rightarrow \operatorname{Aut}\left(\mathbb{C}\left[\pi_{1}(M)\right] \otimes_{\mathbb{C}\left[\pi_{1}\left(M_{G}\right)\right]} \mathbb{C}\right) \\
a & \mapsto(p \otimes v \mapsto a p \otimes v) .
\end{aligned}
$$

In our case $G=\mathbb{Z} / k$ and one can easily see that $\alpha=\alpha_{(z, \chi)}$. Since $\mathbb{Z} / k$ is abelian it follows that $\alpha(G)=\bigoplus_{i=1}^{k} \alpha_{i}$ where $\alpha_{i}: \pi_{1}\left(M_{K}\right) \rightarrow U(1)$ is given by $\alpha_{j}(z):=e^{2 \pi i j / k}$. The proposition now follows from lemmas 2.2 and 3.3 .

We say that a knot $K \subset S^{3}$ has zero Casson-Gordon obstruction if for any prime power $k$ there exists a metabolizer $P_{k} \subset T H_{1}\left(M_{k}\right)$ for the linking pairing such that for any prime power $m$ and $\chi: T H_{1}\left(M_{k}\right) \rightarrow C_{m}$ with $\chi\left(P_{k}\right) \equiv 0$ we get $\tau(K, \chi)=0 \in L_{0}\left(F_{\chi}(t)\right) \otimes \mathbb{Q}$.

The following is an immediate consequence of propositions 5.3 and 5.4 .

Theorem 5.5 Let $K$ be an algebraically slice knot. Then $K$ has zero $S E-$ obstruction if and only if $K$ has zero Casson-Gordon obstruction. 


\section{The Cochran-Orr-Teichner-sliceness obstruction}

\subsection{The Cochran-Orr-Teichner-sliceness filtration}

We give a short introduction to the sliceness filtration introduced by Cochran, Orr and Teichner COT03. For a manifold $W$ denote by $W^{(n)}$ the cover corresponding to $\pi_{1}(W)^{(n)}$. Denote the equivariant intersection form

$$
H_{2}\left(W^{(n)}\right) \times H_{2}\left(W^{(n)}\right) \rightarrow \mathbb{Z}\left[\pi_{1}(W) / \pi_{1}(W)^{(n)}\right]
$$

by $\lambda_{n}$, and the self-intersection form by $\mu_{n}$. An $(n)$-Lagrangian is a submodule $L \subset H_{2}\left(W^{(n)}\right)$ on which $\lambda_{n}$ and $\mu_{n}$ vanish and which maps onto a Lagrangian of $\lambda_{0}: H_{2}(W) \times H_{2}(W) \rightarrow \mathbb{Z}$.

Definition [COT03, def. 8.5] A knot $K$ is called $(n)$-solvable if $M_{K}$ bounds a spin 4-manifold $W$ such that $H_{1}\left(M_{K}\right) \rightarrow H_{1}(W)$ is an isomorphism and such that $W$ admits two dual $(n)$-Lagrangians. This means that $\lambda_{n}$ pairs the two Lagrangians non-singularly and that the projections freely generate $H_{2}(W)$.

A knot $K$ is called (n.5)-solvable if $M_{K}$ bounds a spin 4-manifold $W$ such that $H_{1}\left(M_{K}\right) \rightarrow H_{1}(W)$ is an isomorphism and such that $W$ admits an $(n)$ Lagrangian and a dual $(n+1)$-Lagrangian.

$W$ is called an $(n)$-solution respectively an (n.5)-solution for $K$.

\section{Remark}

(1) The size of an $(n)$-Lagrangian depends only on the size of $H_{2}(W)$, in particular if $K$ is slice, $D$ a slice disk, then $N_{D}$ is an $(n)$-solution for $K$ for all $n$, since $H_{2}\left(N_{D}\right)=0$.

(2) By the naturality of covering spaces and homology with twisted coefficients it follows that if $K$ is $(h)$-solvable, then $K$ is $(k)$-solvable for all $k<h$.

\section{Theorem 6.1}

(1) The following are equivalent.

(a) $K$ is (0)-solvable.

(b) $\operatorname{Arf}(K)=0$.

(b) $\Delta_{K}(t) \equiv \pm 1 \bmod 8$.

(2) A knot $K$ is (0.5)-solvable if and only if $K$ is algebraically slice. 
(3) If $K$ is (1.5)-solvable then $K$ is algebraically slice and $K$ has vanishing Casson-Gordon invariants.

(4) There exist algebraically slice knots which have zero Casson-Gordon invariants but are not (1.5)-solvable.

(5) There exist (2.0)-solvable knots which are not slice.

Statements (1), (2), 3) and (5) are shown in COT03. Taehee Kim Kim02 showed that there exist (1.0)-solvable knots which have zero Casson-Gordon invariants, but are not (1.5)-solvable (cf. also proposition [7.11).

For any $n \in \mathbb{N}$ Tim Cochran and Peter Teicher have examples (unpublished) of knots that are $(n)$-solvable but not $(n .5)$-solvable, in particular are not slice. It is unknown whether for $n \in \mathbb{N}_{0}$ every (n.5)-solvable knot is $(n+1)$-solvable.

\section{2 $\quad L^{2}-$ eta invariants as sliceness-obstructions}

In this section we very quickly summarize some $L^{2}$-eta invariant theory.

Let $M^{3}$ be a smooth manifold and $\varphi: \pi_{1}(M) \rightarrow G$ a homomorphism, then Cheeger and Gromov [ChG85] defined an invariant $\eta^{(2)}(M, \varphi) \in \mathbf{R}$, the (reduced) $L^{2}$-eta invariant. When it is clear which homomorphism we mean, we will write $\eta^{(2)}(M, G)$ for $\eta^{(2)}(M, \varphi)$.

Remark If $\partial(W, \psi)=\left(M^{3}, \varphi\right)$, then (cf. COT03, lemma 5.9 and remark 5.10], LS03])

$$
\eta^{(2)}(M, \varphi)=\operatorname{sign}^{(2)}(W, \psi)-\operatorname{sign}(W),
$$

where $\operatorname{sign}^{(2)}(W, \psi)$ denotes Atiyah's [A76] $L^{2}$-signature.

Let $\mathbb{Q} \Lambda:=\mathbb{Q}\left[t, t^{-1}\right]$.

\section{Theorem 6.2 COT03}

(1) If $K$ is $(0.5)$-solvable, then $\eta^{(2)}\left(M_{K}, \mathbb{Z}\right)=0$.

(2) If $K$ is (1.5)-solvable, then there exists a metabolizer $P_{\mathbb{Q}} \subset H_{1}\left(M_{K}, \mathbb{Q} \Lambda\right)$ for the Blanchfield pairing

$$
\lambda_{B l, \mathbb{Q}}: H_{1}\left(M_{K}, \mathbb{Q} \Lambda\right) \times H_{1}\left(M_{K}, \mathbb{Q} \Lambda\right) \rightarrow \mathbb{Q}(t) / \mathbb{Q}\left[t, t^{-1}\right]
$$

such that for all $x \in P_{\mathbb{Q}}$ we get $\eta^{(2)}\left(M_{K}, \beta_{x}\right)=0$, where $\beta_{x}$ denotes the map

$$
\begin{aligned}
\pi_{1}\left(M_{K}\right) \rightarrow \mathbb{Z} \ltimes H_{1}\left(M_{K}, \Lambda\right) \stackrel{\mathbb{Z} \ltimes H_{1}\left(M_{K}, \mathbb{Q} \Lambda\right)}{ } \underset{ }{\stackrel{i d \times \lambda_{B l, \mathbb{Q}}(x,-)}{\longrightarrow}} \mathbb{Z} \ltimes \mathbb{Q}(t) / \mathbb{Q}\left[t, t^{-1}\right] .
\end{aligned}
$$


We say that a knot $K$ has zero abelian $L^{2}$-eta invariant sliceness obstruction if $\eta^{(2)}\left(M_{K}, \mathbb{Z}\right)=0$ and $K$ has zero metabelian $L^{2}$-eta invariant sliceness obstruction if there exists a metabolizer $P_{\mathbb{Q}} \subset H_{1}\left(M_{K}, \mathbb{Q} \Lambda\right)$ for $\lambda_{B l, \mathbb{Q}}$ such that for all $x \in P_{\mathbb{Q}}$ we get $\eta^{(2)}\left(M_{K}, \beta_{x}\right)=0$. Note that if $K$ has zero metabelian $L^{2}$-eta invariant then it is easy to see that the integral Blanchfield form is metabolic as well and hence $K$ is algebraically slice by proposition 2.1 .

\section{$7 \quad$ Examples}

In this section we will construct

(1) a knot which has zero abelian $L^{2}$-eta invariant, but is not algebraically slice,

(2) a (1.0)-solvable knot which has zero metabelian $L^{2}$-eta invariant, but non-zero SE-obstruction,

(3) a knot which has zero $S T E$-obstruction but non-zero metabelian $L^{2}$-eta invariant (following Taehee Kim Kim02]).

The idea in examples (2) and (3) as well as in the examples of section 8.5 is to start out with a slice knot $K$ and make 'slight' changes via a satellite construction. The change in the eta invariants can be computed explicitly.

\subsection{Satellite knots}

Let $K, C \in S^{3}$ be knots. Let $A \subset S^{3} \backslash K$ a simple closed curve, unknotted in $S^{3}$, note that $S^{3} \backslash N(A)$ is a torus. Let $\varphi: \partial(N(A)) \rightarrow \partial(N(C))$ be a diffeomorphism which sends a meridian of $A$ to a longitude of $C$ and a longitude of $A$ to a meridian of $C$. The space

$$
\overline{S^{3} \backslash N(A)} \cup_{\varphi} \overline{S^{3} \backslash N(C)}
$$

is a 3 -sphere and the image of $K$ is denoted by $S=S(K, C, A)$. We say $S$ is the satellite knot with companion $C$, orbit $K$ and axis $A$. Note that this construction is equivalent to replacing a tubular neighborhood of $C$ by the torus knot $K \subset \overline{S^{3} \backslash N(A)}$.

Proposition 7.1 If $C$ is slice, then for any $K$ and $A$ the satellite knot $S(K, C, A)$ is concordant to $K$. If $K \subset S^{1} \times D^{2} \subset S^{3}$ and $C$ are ribbon, then any $S(K, C, A)$ is in fact ribbon. 
Proof Let $K \subset S^{1} \times D^{2} \subset S^{3}$ and let $C$ be a slice knot. Let $\phi: S^{1} \times I \rightarrow S^{3} \times I$ be a null-concordance for $C$, i.e. $\phi\left(S^{1} \times 0\right)=C$ and $\phi\left(S^{1} \times 1\right)$ is the unknot. We can extend this to a map $\phi: S^{1} \times D^{2} \times I \rightarrow S^{3} \times I$ such that $\phi: S^{1} \times D^{2} \times 0$ is the zero-framing for $C$.

Pick a diffeomorphism $f: \overline{S^{3} \backslash N(A)} \rightarrow S^{1} \times D^{2}$ such that the meridian and longitude of $A$ get sent to the longitude and meridian of $S^{1} \times 0$. Now consider

$$
\psi: S^{1} \times I \rightarrow K \times I \hookrightarrow \overline{S^{3} \backslash N(A)} \times I \stackrel{f \times i d}{\longrightarrow} S^{1} \times D^{2} \times I \stackrel{\phi}{\longrightarrow} S^{3} \times I .
$$

Note that $\phi: S^{1} \times D^{2} \times 1$ is a zero framing for the unknot, since linking numbers are concordance invariants and $\phi: S^{1} \times D^{2} \times 0$ is the zero framing for $C$. This shows that $\psi: S^{1} \times 1 \rightarrow S^{3} \times 1$ gives the satellite knot of the unknot with orbit $K$, i.e. $K$ itself. Therefore $\psi$ gives a concordance between $S=\psi\left(S^{1} \times 0\right)$ and $K=\psi\left(S^{1} \times 1\right)$.

Now assume that $K, C$ are ribbon. Then we can find a concordance $\phi$ which has no minima under the projection $S^{1} \times[0,1] \rightarrow S^{3} \times[0,1] \rightarrow[0,1]$. It is clear that $\psi$ also has no minima, capping off with a ribbon disk for $K$ we get a disk bounding $S$ with no minima, i.e. $S$ is ribbon.

Proposition 7.2 COT04 p. 8] Let $K$ be an (n.0)-solvable knot, $C$ a (0)solvable knot, $A \subset S^{3} \backslash K$ such that $A$ is the unknot in $S^{3}$ and $[A] \in \pi_{1}\left(S^{3} \backslash\right.$ $K)^{(n)}$. Then $S=S(K, C, A)$ is $(n)$-solvable.

\subsection{Eta invariants of satellite knots}

Let $S$ be a satellite knot with companion $C$, orbit $K$ and axis $A$.

Proposition 7.3 [Lit84, p. 337] If $A \in \pi_{1}\left(S^{3} \backslash K\right)^{(1)}$ then the inclusions

$S^{3} \backslash N(K) \hookrightarrow S^{3} \backslash N(K) \backslash N(C) \hookleftarrow\left(\overline{S^{3} \backslash N(A)} \cup_{\varphi} \overline{S^{3} \backslash N(C)}\right) \backslash N(K)=S^{3} \backslash N(S)$

induce isometries of the Blanchfield pairing and the linking pairing of $S$ and $K$ for any cover such that $H_{1}\left(L_{K, k}\right)$ is finite.

This lemma shows in particular that we can identify the set of characters on $H_{1}\left(L_{K, k}\right)$ with the set of characters on $H_{1}\left(L_{S, k}\right)$. The following corollary follows immediately from $\operatorname{Tr} 73$.

Corollary 7.4 If $S=S(K, C, A)$ as above, then $S$ and $C$ have $S$-equivalent Seifert matrices. 
Let $S=S(K, C, A)$ be a satellite knot with $A \in \pi_{1}\left(S^{3} \backslash K\right)^{(1)}$. Let $k$ be any number such that $H_{1}\left(L_{K, k}\right)$ is finite. The curve $A \subset S^{3} \backslash N(K)$ is nullhomologous and therefore lifts to simple closed curves $\tilde{A}_{1}, \ldots, \tilde{A}_{k} \in L_{K, k}$.

Theorem 7.5 Let $z \in S^{1}$ and $\chi: H_{1}\left(L_{K, k}\right)=H_{1}\left(L_{S, k}\right) \rightarrow S^{1}$ a character. Then

$$
\eta\left(M_{S}, \alpha_{(\chi, z)}^{S}\right)=\eta\left(M_{K}, \alpha_{(\chi, z)}^{K}\right)+\sum_{i=1}^{k} \eta\left(M_{C}, \alpha_{i}\right),
$$

where $\alpha_{i}$ denotes the representation $\pi_{1}\left(M_{C}\right) \rightarrow U(1)$ given by $g \mapsto \chi\left(\tilde{A}_{i}\right)^{\epsilon(g)}$.

Proof Litherland Lit84 proved a general statement how to compute the Casson-Gordon invariant of $S$ in terms of the Casson-Gordon invariant of $K$ and the basic invariants of $C$. Translating the proof into the language of eta invariants shows that

$$
\eta\left(M_{S, k}, \beta_{(\chi, z)}^{S}\right)=\eta\left(M_{K, k}, \beta_{(\chi, z)}^{K}\right)+\sum_{i=1}^{k} \eta\left(M_{C}, \alpha_{i}\right) .
$$

For full details we refer to [Fr03d]. Casson-Gordon invariants only make sense when $k$ and the order of $\chi$ are prime powers, but the proof can be used to show the above statement about eta invariants.

By corollary 7.4 the knots $K$ and $S$ have $S$-equivalent Seifert matrices, in particular the twisted signatures are the same (cf. proposition 3.3). The theorem now follows immediately from proposition 5.4.

Iterating the satellite construction we can generalize the theorem as follows (cf. Liv02, p. 405]). Let $K \in S^{3}$ be a knot and $A_{1}, \ldots, A_{s} \in S^{3} \backslash K$ be simple closed curves which form the unlink in $S^{3}$ and such that $A_{i}=0 \in \pi_{1}\left(S^{3} \backslash K\right)^{(1)}$. Let $C_{1}, \ldots, C_{s}$ be knots. Then we can inductively form satellite knots by setting $S_{0}:=K$ and $S_{i}$ the satellite formed with orbit $S_{i-1}$, companion $C_{i}$ and axis $A_{i}$. Note that $A_{i} \in \pi_{1}\left(S^{3} \backslash S_{i-1}\right)^{(1)}$. We write

$$
S_{i}=: S\left(K, C_{1}, \ldots, C_{i}, A_{1}, \ldots, A_{i}\right) .
$$

Theorem 7.6 Let $S:=S\left(K, C_{1}, \ldots, C_{s}, A_{1}, \ldots, A_{s}\right)$ as above. Let $k$ be any number such that $H_{1}\left(L_{K, k}\right)$ is finite, $z \in S^{1}$ and $\chi: H_{1}\left(L_{S, k}\right) \rightarrow S^{1}$ a character, denote the corresponding character $H_{1}\left(L_{K, k}\right) \rightarrow S^{1}$ by $\chi$ as well. Then

$$
\eta\left(M_{S}, \alpha_{(\chi, z)}^{S}\right)=\eta\left(M_{K}, \alpha_{(\chi, z)}^{K}\right)+\sum_{j=1}^{s} \sum_{i=1}^{k} \eta\left(M_{C_{j}}, \alpha_{i j}\right) .
$$

Here $\alpha_{i j}$ denotes the representation $\pi_{1}\left(M_{C_{j}}\right) \rightarrow U(1)$ given by $g \mapsto \chi\left(\left(\tilde{A}_{j}\right)_{i}\right)^{\epsilon(g)}$, where $\left(\tilde{A}_{j}\right)_{1}, \ldots,\left(\tilde{A}_{j}\right)_{k}$ denote the lifts of $A_{j}$ to $L_{S, k}$. 


\subsection{Computation of $L^{2}$-eta invariants}

The following proposition makes it possible to compute $L^{2}$-eta invariants in many cases.

Proposition 7.7 ( COT03, Fr03c] Let $K$ be a knot, then

$$
\eta^{(2)}\left(M_{K}, \mathbb{Z}\right)=\int_{S^{1}} \sigma_{z}(K)
$$

We quote a theorem by Cochran, Orr and Teichner on the computation of $L^{2}$-eta invariants for satellite knots. Recall that for $x \in H_{1}(M, \Lambda)$ we defined

$$
\begin{aligned}
& \beta_{x}: \pi_{1}\left(M_{K}\right) \rightarrow \mathbb{Z} \ltimes H_{1}\left(M_{K}, \Lambda\right) \rightarrow \mathbb{Z} \ltimes H_{1}\left(M_{K}, \mathbb{Q} \Lambda\right) \\
& \stackrel{i d \times \lambda_{B l, \mathbb{Q}}(x,-)}{\longrightarrow} \mathbb{Z} \ltimes \mathbb{Q}(t) / \mathbb{Q}\left[t, t^{-1}\right] .
\end{aligned}
$$

Theorem 7.8 ([COT04 p. 8] [Kim02, prop. 5.3]) Let $S=S(K, C, A)$ with $A \in \pi_{1}\left(S^{3} \backslash K\right)^{(1)}$. Let $x \in H_{1}\left(M_{S}, \Lambda\right)=H_{1}\left(M_{K}, \Lambda\right)$, then

$$
\eta^{(2)}\left(M_{S}, \beta_{x}\right)= \begin{cases}\eta^{(2)}\left(M_{K}, \beta_{x}\right)+\eta^{(2)}\left(M_{C}, \mathbb{Z}\right) & \text { if } \beta_{x}(A) \neq 0 \\ \eta^{(2)}\left(M_{K}, \beta_{x}\right) & \text { if } \beta_{x}(A)=0\end{cases}
$$

where $A$ is considered as an element in $H_{1}\left(M_{K}, \Lambda\right)$.

In [Fr03c] we give a different approach to the computation of metabelian $L^{2}$-eta invariants based on an approximation theorem by Lück and Schick [LS01].

\subsection{Examples}

Let

$$
B_{1}:=\left(\begin{array}{cccc}
0 & 0 & 1 & 1 \\
0 & 0 & 0 & 1 \\
1 & 1 & 0 & 1 \\
0 & 1 & 0 & 0
\end{array}\right)
$$

this Seifert matrix is obviously metabolic. The Alexander polynomial is $\Delta_{B_{1}}(t)$ $=\left(t^{2}-t+1\right)^{2}$. The signature function $z \mapsto \sigma_{z}\left(B_{1}\right)$ is zero outside of the set of zeros of the Alexander polynomial since the form is metabolic. The zeros are $e^{2 \pi i / 6}, e^{2 \pi 5 i / 6}$ and at both points the signature is -1 . Let

$$
B_{2}:=\left(\begin{array}{ll}
1 & 1 \\
0 & 1
\end{array}\right)
$$


Then $\Delta_{B_{2}}(t)=t^{2}-t+1$, and

$$
\sigma_{e^{2 \pi i t}}\left(B_{2}\right)= \begin{cases}2 & \text { for } t \in\left(\frac{1}{6}, \frac{5}{6}\right) \\ 0 & \text { for } t \in\left[0, \frac{1}{6}\right) \cup\left(\frac{5}{6}, 1\right]\end{cases}
$$

Finally let

$$
B_{3}:=\left(\begin{array}{cccccc}
1 & 0 & 0 & 0 & 1 & 0 \\
-1 & 1 & 0 & 1 & 0 & 1 \\
0 & 0 & 0 & 1 & 1 & 1 \\
0 & 1 & 0 & 1 & 0 & 1 \\
1 & 0 & 1 & 0 & 1 & 1 \\
0 & 1 & 1 & 1 & 0 & 1
\end{array}\right)
$$

Then $\Delta_{B_{3}}(t)=\Phi_{14}(t)=1-t+t^{2}-t^{3}+t^{4}-t^{5}+t^{6}$, and

$$
\sigma_{e^{2 \pi i t}}\left(B_{3}\right)= \begin{cases}2 & \text { for } t \in\left(\frac{1}{14}, \frac{3}{14}\right) \cup\left(\frac{5}{14}, \frac{9}{14}\right) \cup\left(\frac{11}{14}, \frac{13}{14}\right) \\ 0 & \text { for } t \in\left[0, \frac{1}{14}\right) \cup\left(\frac{3}{14}, \frac{5}{14}\right) \cup\left(\frac{9}{14}, \frac{11}{14}\right) \cup\left(\frac{13}{14}, 1\right]\end{cases}
$$

Proposition 7.9 (Example 1) There exists a (0)-solvable knot $K$ with zero abelian $L^{2}$-eta invariant but which is not algebraically torsion.

Proof Recall that for a knot $\operatorname{Arf}(K)=0$ if and only if $\Delta_{K}(-1) \equiv \pm 1$ mod 8. By Lev69] we can find a knot $K$ with Seifert matrix $7 B_{3} \oplus-6 B_{2}$, then $\operatorname{Arf}(K)=7 \operatorname{Arf}\left(B_{3}\right)-6 \operatorname{Arf}\left(B_{2}\right)=0$, using the above calculations we get $\sigma_{z}(K)=2$ for $z=e^{2 \pi i k / 5}, k=1,2,3,4$ and $\int_{S^{1}} \sigma_{z}(K)=0$. This shows that $K$ has all the required properties.

Proposition 7.10 (Example 2) There exists a (1.0)-solvable knot $K$ with zero metabelian $L^{2}$-eta invariant, but non-zero SE-invariants.

Proof Let $p(t)=-2 t+5-2 t^{-1}$. By Kearton Kea73 there exists a knot $K_{5} \cong S^{5} \subset S^{7}$ such that its Blanchfield pairing is isomorphic to

$$
\begin{aligned}
\Lambda / p(t)^{2} \times \Lambda / p(t)^{2} & \rightarrow S^{-1} \Lambda / \Lambda \\
(a, b) & \mapsto \bar{a} p(t)^{-2} b
\end{aligned}
$$

Let $A$ be a Seifert matrix for $K_{5}$, and $K \subset S^{3}$ be a slice knot with Seifert matrix $A$. Since the Blanchfield pairing is determined by $A$, the Blanchfield pairing of $K$ is isomorphic to the Blanchfield pairing of $K_{5}$. A computation using lemma 2.2 shows that $\left|H_{1}\left(L_{K, 4}\right)\right|=225$.

Let $N$ be a non-negative integer greater or equal than $X:=\max \left\{\eta\left(M_{K}, \alpha\right) \mid \alpha \in\right.$ $\left.R_{4}\left(\pi_{1}\left(M_{K}\right)\right)\right\}$. Note that $R_{4}\left(\pi_{1}\left(M_{K}\right)\right)$ is compact since $\pi_{1}\left(M_{K}\right)$ is finitely 
generated. Using of results of Levine [Lev94, p. 92] one can show that $X$ is finite (cf. Fr03c for details).

Let $F$ be a Seifert surface for $K$. We can view $F$ as a disk with $2 g$ 1-handles attached. Then the meridians $A_{1}, \ldots, A_{2 g} \in S^{3} \backslash F$ of the handles form the unlink in $S^{3}$ and the corresponding homology classes give a basis for $H_{1}\left(S^{3} \backslash F\right)$. Note that $A_{i} \in \pi_{1}\left(S^{3} \backslash K\right)^{(1)}$. Denote the knot of the proof of proposition 7.9 by $D$ and let $C=(N+1) \cdot D$, and form the iterated satellite knot

$$
S:=S\left(K, C, \ldots, C, A_{1}, \ldots, A_{2 g}\right) .
$$

We claim that the satellite knot $S$ satisfies the conditions stated in the proposition. $S$ is (1.0)-solvable by theorem $\mathbf{7 . 2}$ and has zero metabelian $L^{2}$-eta invariant by theorem 7.8 since $K$ is slice, $\int_{S^{1}} \sigma_{z}(C)=0$ by construction of $C$ and since $K$, and therefore also $S$, has a unique metabolizer for the Blanchfield pairing.

We have to show that for all $P_{4} \subset H_{1}\left(L_{S, 4}\right)$ with $P_{4}=P_{4}^{\perp}$ with respect to the linking pairing $\lambda_{S, 4}$, we can find a non-zero character $\chi: H_{1}\left(L_{S, 4}\right) \rightarrow S^{1}$ of prime power order, vanishing on $P_{4}$, such that for one transcendental $z$ we get $\eta\left(M_{S}, \alpha_{(z, \chi)}\right) \neq 0$.

Let $P_{4}$ be a metabolizer and $\chi: H_{1}\left(L_{S, 4}\right) \rightarrow S^{1}$ a non-trivial character of order 5 , vanishing on $P_{4}$. Denote the corresponding character on $H_{1}\left(L_{K, 4}\right)$ by $\chi$ as well. For any $z \in S^{1}$ we get by corollary [7.5

$$
\eta\left(M_{S}, \alpha_{(\chi, z)}^{S}\right)=\eta\left(M_{K}, \alpha_{(\chi, z)}^{K}\right)+\sum_{j=1}^{2 g} \sum_{i=1}^{4} \eta\left(M_{C}, \alpha_{i j}\right),
$$

where $\alpha_{i j}$ denotes the representation $\pi_{1}\left(M_{C}\right) \rightarrow U(1)$ given by $g \mapsto \chi\left(\left(\tilde{A}_{j}\right)_{i}\right)^{\epsilon(g)}$ and $\left(\tilde{A}_{j}\right)_{i}$ denotes the $i^{\text {th }}$ lift of $A_{j}$ to $L_{K, k}$. By definition of $N$ and by proposition 3.3 we get

$$
\begin{aligned}
\eta\left(M_{S}, \alpha_{(\chi, z)}^{S}\right) & \geq-N+\sum_{j=1}^{2 g} \sum_{i=1}^{4} \eta\left(M_{C}, \alpha_{i j}\right) \\
& =-N+\sum_{j=1}^{2 g} \sum_{i=1}^{4} \sigma_{\chi\left(\left(\tilde{A}_{j}\right)_{i}\right)}(C) \\
& =-N+\sum_{j=1}^{2 g} \sum_{i=1}^{4}(N+1) \sigma_{\chi\left(\left(\tilde{A}_{j}\right)_{i}\right)}\left(B_{2}\right)
\end{aligned}
$$

Note that $\eta\left(M_{C}, \alpha_{i j}\right) \geq 0$ for all $i, j$ since $\sigma_{e^{2 \pi i j / 5}}(C) \geq 0$ for $j=0, \ldots, 4$. The lifts $\left(\tilde{A}_{j}\right)_{i}$ are easily seen to generate $H_{1}\left(L_{K, 4}\right)$, hence $\chi\left(\left(\tilde{A}_{j}\right)_{i}\right) \neq 1$ for at least one $(i, j)$ since $\chi$ is non-trivial. But $\sigma_{w}(C)=2(N+1)$ for $w=e^{2 \pi i j / 5}, j=$ $1,2,3,4$. It follows that $\eta\left(M_{S}, \alpha_{(\chi, z)}^{S}\right) \geq-N+2(N+1)>0$ for all $z$.

For completeness sake we add the following example which was discovered by Taehee Kim Kim02. 
Proposition 7.11 (Example 3) There exists a knot $S$ which is algebraically slice, (1.0)-solvable, has zero STE-obstruction but non-zero metabelian $L^{2}$-eta invariant.

Proof Denote by $\Phi_{30}(t)=1+t-t^{3}-t^{4}-t^{5}+t^{7}+t^{8}$ the minimal polynomial of $e^{2 \pi i / 30}$. As in the proof of proposition 7.10 there exists a ribbon knot $K$ such that the Blanchfield pairing is isomorphic to

$$
\begin{aligned}
\Lambda / \Phi_{30}(t)^{2} \times \Lambda / \Phi_{30}(t)^{2} & \rightarrow S^{-1} \Lambda / \Lambda \\
(a, b) & \mapsto \bar{a} \Phi_{30}(t)^{-2} b
\end{aligned}
$$

An explicit example of such a knot is given by Taehee Kim [Kim02, Section 2]. Note that $K$ has a unique metabolizer $P$ for the Blanchfield pairing. Furthermore $H_{1}\left(L_{K, k}\right)=0$ for all prime powers $k$ by theorem 4.9. Let $C$ be a knot with Seifert matrix $B_{1}$ and $A \in \pi_{1}\left(S^{3} \backslash K\right)^{(1)}$ unknotted in $S^{3}$ and such that $\beta_{x}(A) \neq 0$ for some $x \in P$.

By corollary 7.4 the knot $S$ is algebraically slice, since $K$ is algebraically slice. $S$ is (1.0)-solvable by proposition 7.2 since $\operatorname{Arf}(C)=0$.

Since $H_{1}\left(L_{S, k}\right)=H_{1}\left(L_{K, k}\right)=0$ for all prime powers $k$, we get $R_{k}^{i r r, m e t}\left(\pi_{1}\left(M_{K}\right)\right)$ $=\emptyset$ for all prime powers $k$, hence $S$ has zero STE-obstruction. By theorem 7.8 $S$ has non-zero metabelian $L^{2}$-eta invariant since $\beta_{x}(S)=\beta_{x}(K)+\int_{S^{1}} \sigma_{z}(C)=$ $\frac{4}{3}$ and $P$ is the unique metabolizer.

\section{Ribbon knots, doubly slice knots and the obstruc- tions of Gilmer and Letsche}

\subsection{Obstructions to a knot being ribbon}

An immersed ribbon disk is an immersion $D \rightarrow S^{3}$ which bounds $K$ such that the singularities are only of the type as in figure 1, i.e. the self-intersection lies completely in the interior of one of the two sheets involved. The singularities can be resolved in $D^{4}$ to give an embedded slice disk. Slice disks which are isotopic to such disks are called ribbon disks. If a knot has a ribbon disk we say that the knot is ribbon. Note that this is a purely 3-dimensional definition.

It is a longstanding conjecture of Fox (cf. [Fox61, problem 25]) that all slice knots are ribbon. In theorem 8.3 we give a condition for a knot to be ribbon which is ostensibly stronger than the corresponding condition (theorem 4.7) for a knot to be slice. It is an intriguing question whether one can use these results to disprove Fox's conjecture. 


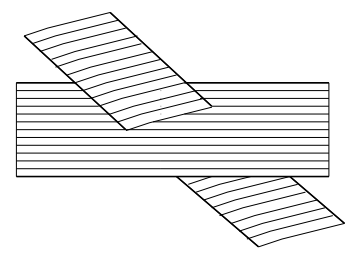

Figure 1: Immersed ribbon disk.

Proposition 8.1 (Gor81, lemma 3.1] [Kea75b, lemma 2.1]) If $K$ is ribbon and $D \subset D^{4}$ a ribbon disk, then the maps

$$
\begin{aligned}
i_{*}: \pi_{1}\left(S^{3} \backslash K\right) & \rightarrow \pi_{1}\left(D^{4} \backslash D\right) \\
H_{1}\left(M_{K}, \Lambda\right) & \rightarrow H_{1}\left(N_{D}, \Lambda\right)
\end{aligned}
$$

are surjective.

Proposition 8.2 Assume that $K$ is ribbon, $D$ a ribbon disk, then $H_{1}\left(N_{D}, \Lambda\right)$ is $\mathbb{Z}$-torsion free, in particular $P:=\operatorname{Ker}\left\{H_{1}\left(M_{K}, \Lambda\right) \rightarrow H_{1}\left(N_{D}, \Lambda\right)\right\}$ is a metabolizer for $\lambda_{B l}$.

Proof According to [Lev77, thm. 2.1 and prop. 2.4] there exists a short exact sequence

$$
0 \rightarrow \operatorname{Ext}_{\Lambda}^{2}\left(H_{1}(N, M, \Lambda)\right) \rightarrow \overline{H_{1}(N, \Lambda)} \rightarrow \operatorname{Ext}_{\Lambda}^{1}\left(H_{2}(N, M, \Lambda)\right) \rightarrow 0 .
$$

Here $\overline{H_{1}(N, \Lambda)}$ denotes $H_{1}(N, \Lambda)$ with involuted $\Lambda$-module structure, i.e. $t \cdot v:=$ $t^{-1} v$. Furthermore $\operatorname{Ext}_{\Lambda}^{1}\left(H_{2}(N, M, \Lambda)\right)$ is $\mathbb{Z}$-torsion free (cf. [Lev77, prop. 3.2]). In order to show that $H_{1}(N, \Lambda)$ is $\mathbb{Z}$-torsion free it is therefore enough to show that $H_{1}(N, M, \Lambda)=0$. Consider the exact sequence

$$
H_{1}(M, \Lambda) \rightarrow H_{1}(N, \Lambda) \rightarrow H_{1}(N, M, \Lambda) \rightarrow H_{0}(M, \Lambda) \rightarrow H_{0}(N, \Lambda) \rightarrow 0 .
$$

The last map is an isomorphism. By proposition 8.1 the first map is surjective. It follows that $H_{1}(N, M, \Lambda)=0$.

The second part follows immediately from theorem 2.1 .

Theorem 8.3 Let $K \subset S^{3}$ be a ribbon knot. Then there exists a metabolizer $P \subset H_{1}\left(M_{K}, \Lambda\right)$ such that for any $\alpha \in P_{k}^{i r r}\left(\pi_{1}\left(M_{K}\right)\right)$ vanishing on $0 \times P$ we get $\eta\left(M_{k}, \alpha\right)=0$.

Proof Let $P:=\operatorname{Ker}\left\{H_{1}\left(M_{K}, \Lambda\right) \rightarrow H_{1}\left(N_{D}, \Lambda\right)\right\}$ where $D$ is a ribbon disk for $K$. Then $P=P^{\perp}$ by proposition 8.2, Let $\alpha \in P_{k}\left(\pi_{1}(M)\right)$ which vanishes on $0 \times P$, then $\alpha$ extends to a metabelian representation of $\pi_{1}\left(N_{D}\right)$, hence $\eta\left(M_{K}, \alpha_{(z, \chi)}\right)=0$ by lemma 4.3 and theorem 4.4 
We say that $K$ has zero eta invariant ribbonness obstruction if the conclusion holds for $K$.

Remark One can show that if $P$ is a metabolizer for $\lambda_{B l}$ and $k$ is such that $H_{1}\left(L_{k}\right)$ is finite, then $P_{k}:=\pi_{k}(P) \subset T H_{1}\left(M_{k}\right)=H_{1}(M, \Lambda) /\left(t^{k}-1\right)$ is a metabolizer for $\lambda_{L, k}$. In particular if $K$ is and $k$ any number such that $H_{1}\left(L_{k}\right)$ is finite then there exists a metabolizer $P_{k}$ for the linking pairing such that for all $\chi: T H_{1}\left(M_{k}\right) \rightarrow S^{1}$ of prime power order, vanishing on $P_{k}$, and for all transcendental $z \in S^{1}$ we get $\eta\left(M_{K}, \alpha_{(z, \chi)}\right)=0$.

Comparing this result with theorem 4.7 we see that the ribbon obstruction is stronger in two respects. When $K$ is ribbon

(1) we can find metabolizers $P_{k}$ which all lift to the same metabolizer of the Blanchfield pairing,

(2) the representations for non-prime power dimensions don't have to be tensor products.

We will make use of this in proposition 8.9.

Remark (1) Note that the only fact we used was that for a ribbon disk $H_{1}\left(M_{K}, \Lambda\right) \rightarrow H_{1}\left(N_{D}, \Lambda\right)$ is surjective.

(2) Casson and Gordon [CG86, p. 154] prove a ribbon-obstruction theorem which does not require the character to be of prime power order, but has a strong restriction on the fundamental group of $\pi_{1}\left(N_{D}\right)$.

(3) In Fr03c we show that if a knot $K$ has zero eta invariant ribbonness obstruction, then $K$ has in particular zero metabelian $L^{2}$-eta invariant sliceness obstruction. In proposition 8.9 we will see that the converse is not true.

\subsection{Obstructions to a knot being doubly slice}

A knot $K \subset S^{3}$ is called doubly slice (or doubly null-concordant) if there exists an unknotted smooth two-sphere $S \subset S^{4}$ such that $S \cap S^{3}=K$. It follows from the Schoenflies theorem that a doubly slice knot is in particular slice. Fox Fox61 posed the question which slice knots are doubly slice. Doubly slice knots have been studied by Sumners [S71, Levine Lev89, Ruberman Ru83. and Taehee Kim Kim02.

We say that knot $K$ is algebraically doubly slice if $K$ has a Seifert matrix of the form $\left(\begin{array}{cc}0 & B \\ C & 0\end{array}\right)$ where $B, C$ are square matrices of the same size. Sumners 
S71 showed that if $K \subset S^{3}$ is doubly slice, then $K$ is algebraically doubly slice. This result can be used to show that many slice knots are not doubly slice.

We prove the following new doubly sliceness obstruction theorem.

Theorem 8.4 Let $K \subset S^{3}$ be a doubly slice knot. Then there exist metabolizers $P_{1}, P_{2} \subset H_{1}\left(M_{K}, \Lambda\right)$ for the Blanchfield pairing such that

(1) $H_{1}\left(M_{K}, \Lambda\right)=P_{1} \oplus P_{2}$

(2) for any $\alpha \in P_{k}^{\text {irr }}\left(\mathbb{Z} \ltimes H_{1}\left(M_{K}, \Lambda\right)\right)$ vanishing on $0 \times P_{i}$, i=1,2, we get $\eta\left(M_{K}, \alpha\right)=0$.

Proof Let $S \subset S^{4}$ be an unknotted two-sphere such that $S \cap S^{3}=K$. Intersecting $S$ with $\left\{\left(x_{1}, \ldots, x_{5}\right) \in \mathbf{R}^{5} \mid x_{5} \geq 0\right\}$ and $\left\{\left(x_{1}, \ldots, x_{5}\right) \in \mathbf{R}^{5} \mid x_{5} \leq 0\right\}$ we can write write $S=D_{1}^{2} \cup_{K} D_{2}^{2}$ and $S^{4}=D_{1}^{4} \cup_{S^{3}} D_{2}^{4}$. Let $N_{i}=D_{i}^{4} \backslash D_{i}^{2}$, then $N_{1} \cap N_{2}=S^{3} \backslash K$ and $N_{1} \cup N_{2}=S^{4} \backslash S$. From the Mayer-Vietoris sequence we get

$$
H_{1}\left(M_{K}, \Lambda\right)=H_{1}\left(N_{1}, \Lambda\right) \oplus H_{1}\left(N_{2}, \Lambda\right)
$$

since $H_{1}\left(M_{K}, \Lambda\right)=H_{1}\left(S^{3} \backslash K, \Lambda\right)$ and $H_{1}\left(S^{4} \backslash S, \Lambda\right)=0$ since $S$ is trivial.

Now let $P_{i}:=\operatorname{Ker}\left\{H_{1}(M, \Lambda) \rightarrow H_{1}\left(N_{i}, \Lambda\right)\right\}$, the proof concludes as the proof of the main ribbon obstruction theorem 8.3 since $H_{1}\left(M_{K}, \Lambda\right) \rightarrow H_{1}\left(N_{i}, \Lambda\right)$ is surjective for $i=1,2$.

Remark (1) The proof of theorem 8.4 shows in particular that if $K$ is doubly slice we can find a slice disk $D$ such that $H_{1}\left(N_{D}, \Lambda\right)$ is $\mathbb{Z}$-torsion free.

(2) Comparing theorem 8.4 with theorems 4.7 and 8.3 we see that doubly slice knots have zero (doubly) ribbon obstruction.

Question 8.5 Using the notation of the proof we get from the van Kampen theorem that for a doubly slice knot

$$
\mathbb{Z}=\pi_{1}\left(D_{1}^{4} \backslash D_{1}^{2}\right) *_{\pi_{1}\left(S^{3} \backslash K\right)} \pi_{1}\left(D_{2}^{4} \backslash D_{2}^{2}\right) .
$$

Can we conclude that $\pi_{1}\left(S^{3} \backslash K\right) \rightarrow \pi_{1}\left(D_{i}^{4} \backslash D_{i}^{2}\right)$ is surjective for at least one $i$ ? If yes, then this would show that a doubly slice knot is in fact homotopically ribbon. One can go further and ask whether doubly slice knots are in fact ribbon or doubly ribbon. The first part of the question is of course a weaker version of the famous 'slice equals ribbon' conjecture. 
Remark Taehee Kim Kim03, Kim04 introduced the notion of $(n, m)$-solvability $\left(n, m \in \frac{1}{2} \mathbb{N}\right)$. He used $L^{2}$-eta invariants to find highly non-trivial examples of non doubly slice knots, in particular for each $n \in \mathbb{N}$ he found examples of knots which are $(n, n)$-solvable but not $(n+1, n+1)$-solvable.

\subsection{The Gilmer-obstruction}

In sections 8.3 and 8.4 we quickly recall the obstructions of Gilmer Gi83, Gi93 and Letsche Let00]. The papers claim to define sliceness obstructions. While investigating the precise connection to our obstructions we found that unfortunately the proofs in their paper contain gaps. We show that Gilmer's and Letsche's results give ribbon obstructions and we give a precise description of the problems that must be solved in order to show that they are really sliceness obstructions.

Let $K$ be a knot, $F$ a Seifert surface. Pick a basis $a_{1}, \ldots, a_{2 g}$ for $H_{1}(F)$, denote by $A$ the corresponding Seifert matrix. Let $\Gamma:=\left(A^{t}-A\right)^{-1} A^{t}$ and $k$ such that $H_{1}\left(L_{k}\right)$ is finite. Define $\varphi_{k}: H_{1}(F) \rightarrow H_{1}(F)$ to be the endomorphism given by $\Gamma^{k}-(\Gamma-1)^{k}$ and define $B^{k} \subset H_{1}(F, \mathbb{Q} / \mathbb{Z})$ to be the kernel of $\varphi_{k} \otimes \mathbb{Q} / \mathbb{Z}$. For a prime number $p$ define $B_{p}^{k}$ to be the $p$-primary part of $B^{k}$. Let $Y$ be $S^{3}$ cut along $F$ and denote by $\alpha_{1}, \ldots, \alpha_{2 g} \in H_{1}(Y)$ the dual basis with respect to Alexander duality, i.e. $\operatorname{lk}\left(a_{i}, \alpha_{j}\right)=\delta_{i j}$.

Pick a lift of $Y$ to $L_{k}$. Denote the lifts of $\alpha_{i}$ by $\tilde{\alpha}_{i}$. These generate $H_{1}\left(L_{k}\right)$, in fact

$$
H_{1}\left(L_{k}\right)=\left(\bigoplus \mathbb{Z} \tilde{\alpha}_{i}\right) / \Gamma_{k}^{t} .
$$

We therefore get a well-defined isomorphism

$$
\begin{aligned}
B^{k} & \rightarrow H^{1}\left(L_{k}, \mathbb{Q} / \mathbb{Z}\right)=\operatorname{Hom}\left(H_{1}\left(L_{k}\right), \mathbb{Q} / \mathbb{Z}\right) \\
\sum r_{i} a_{i} & \mapsto\left(\tilde{\alpha}_{j} \mapsto r_{j}\right) \text { where } r_{j} \in \mathbb{Q} / \mathbb{Z} .
\end{aligned}
$$

Different lifts of $Y$ to $L_{k}$ give different characters, but the associated CassonGordon invariants agree. Hence we get a well-defined Casson-Gordon invariant $\tau(K, \chi) \in L_{0}\left(F_{\chi}(t)\right) \otimes \mathbb{Q}$ for $\chi \in B^{k}$.

We say that a knot $K$ has zero Gilmer obstruction for a Seifert surface $F$ and a prime $p$ if there exists a metabolizer $H$ for the Seifert pairing on $H_{1}(F)$ such that for all prime powers $k, \tau\left(K, B_{p}^{k} \cap(H \otimes \mathbb{Q} / \mathbb{Z})\right)=0$. More precisely, for a character $\chi \in B_{p}^{k} \cap(H \otimes \mathbb{Q} / \mathbb{Z})$ we get $\tau(K, \chi)=0 \in L_{0}\left(F_{\chi}(t)\right)$.

Gilmer's theorem (cf. Gi93, p. 5]) claims that a slice knot has zero Gilmer obstruction for all Seifert surfaces and all primes. Unfortunately the proof has 
a gap. On page 6 , the statement that $H \otimes \mathbb{Q} / \mathbb{Z}$ is the kernel of $\mu_{*}$ (Gilmer's notation in the paper) is not necessarily true since tensoring with $\mathbb{Q} / \mathbb{Z}$ is not exact. This becomes a problem if $H_{1}(R)$ is not torsion free, where $R$ denotes a 3 -manifold which bounds the union of $F$ with a slice disk $D$. One can show that the question whether $H_{1}(R)$ is torsion free is closely related to the question whether $\operatorname{Ker}\left\{H_{1}\left(M_{K}, \Lambda\right) \rightarrow H_{1}\left(N_{D}, \Lambda\right)\right\}$ is a metabolizer for the Blanchfield pairing.

Furthermore the proof of the cancellation lemma 5 has a gap as well, namely on the second to last line. Note that the same problem appears in Gilmer's earlier paper Gi83.

Going carefully through the proof of Gilmer's theorem, or the equivalent version in terms of eta invariants in Fr03d] one can see the following.

Theorem 8.6 Let $K$ be a slice knot and $F$ a Seifert surface. Then $K$ has zero Gilmer obstruction for $F$ and all but finitely many primes $p$.

In Fr03d] we show that if a knot satisfies the vanishing condition on eta invariants of theorem 8.3 then the Gilmer obstruction vanishes for all Seifert surfaces and all primes. In particular this shows the following theorem.

Theorem 8.7 Let $K$ be a ribbon knot or a doubly slice knot, then $K$ has zero Gilmer obstruction for all $F$ and all primes.

\subsection{The Letsche--obstruction}

For $x \in H_{1}\left(M_{K}, \Lambda\right)$ we define the map

$$
\begin{aligned}
\alpha_{x}: \pi_{1}\left(M_{K}\right) \rightarrow \pi_{1}\left(M_{K}\right) / \pi_{1}\left(M_{K}\right)^{(2)} & \cong \mathbb{Z} \ltimes H_{1}\left(M_{K}, \Lambda\right) \\
& \stackrel{i d \times \lambda_{B l}(x,-)}{\longrightarrow} \mathbb{Z} \ltimes S^{-1} \Lambda / \Lambda .
\end{aligned}
$$

We say that a knot $K$ has zero Letsche obstruction if there exists a metabolizer $P \subset H_{1}\left(M_{K}, \Lambda\right)$ such that for any $k$, any $x \in P$ and any $\theta \in R_{k}\left(\mathbb{Z} \ltimes S^{-1} \Lambda / \Lambda\right)$ such that $\theta \circ \alpha_{x} \in P_{k}^{i r r}\left(\pi_{1}\left(M_{K}\right)\right)$ we get $\eta\left(M_{K}, \theta \circ \alpha_{x}\right)=0$. Letsche (cf. [Let00, p. 313]) claims that every slice knot has zero Letsche obstruction. Unfortunately the statement of the last paragraph of the proof of lemma 2.21 is incorrect since maps $\chi_{1}, \chi_{2}$ to $S^{1}$ whose $n^{\text {th }}$ powers $\chi_{1}^{n}, \chi_{2}^{n}$ agree for some $n>1$, don't have to be identical, i.e. $\chi_{1} \neq \chi_{2}$. In particular given an abelian group $P$ and a map $\chi: n P \rightarrow S^{1}$ for some $n>1$ there is no canonical way to extend $\chi$ to $P$. 
The situation in Letsche's paper is as follows. Let $D$ be a slice disk for $K$, then by theorem $2.1 P:=\operatorname{Ker}\left\{H_{1}\left(M_{K}, \Lambda\right) \rightarrow F H_{1}\left(N_{D}, \Lambda\right)\right\}$ is a metabolizer, but only characters which vanish on $Q:=\operatorname{Ker}\left\{H_{1}\left(M_{K}, \Lambda\right) \rightarrow H_{1}\left(N_{D}, \Lambda\right)\right\}$ extend over the slice disk complement. Note that $Q=n P$ for some $n \in \mathbb{N}$. If $x \in P$ and $n \neq 1$, then $\alpha_{x}$ does not vanish on the metabolizer $P$ but only on $Q=n P$. Letsche's attempt to get around this problem introduced the above mentioned problem.

On the other hand if $Q=P$, i.e. if $\operatorname{Ker}\left\{H_{1}\left(M_{K}, \Lambda\right) \rightarrow H_{1}\left(N_{D}, \Lambda\right)\right\}$ is a metabolizer for the Blanchfield pairing, then the Letsche obstruction vanishes. In particular we get the following weaker statement.

Theorem 8.8 Let $K \subset S^{3}$ be a slice knot, $D$ a slice disk. If $\operatorname{Ker}\left\{H_{1}\left(M_{K}, \Lambda\right)\right.$ $\left.\rightarrow H_{1}\left(N_{D}, \Lambda\right)\right\}$ is a metabolizer for the Blanchfield pairing, then $K$ has zero Letsche obstruction. In particular ribbon knots and doubly-slice knots have zero Letsche obstruction.

Remark Note that there is no restriction on the dimensions of the representations. In Fr03d we show that all unitary irreducible representations of $\mathbb{Z} \ltimes S^{-1} \Lambda / \Lambda$ are in fact tensor products of unitary representations of prime power dimensions.

We give a complete proof, which differs somewhat from Letsche's original proof.

Proof Let $x \in P$. Considering the long exact sequence we see that $x=$ $\partial(w)$ for some $w \in H_{2}\left(N_{D}, M_{K}, \Lambda\right)$. Letsche [Let00 showed that in fact $H_{2}\left(N_{D}, M_{K}, \Lambda\right)=\operatorname{Tor}_{\Lambda} H_{2}\left(N_{D}, M_{K}, \Lambda\right)$ and that there exists a Blanchfield pairing

$$
\lambda_{B l, N_{D}}: \operatorname{Tor}_{\Lambda} H_{2}\left(N_{D}, M_{K}, \Lambda\right) \times \operatorname{Tor}_{\Lambda} H_{1}\left(N_{D}, \Lambda\right) \rightarrow S^{-1} \Lambda / \Lambda
$$

such that $\lambda_{B l}(x, y)=\lambda_{B l, N_{D}}\left(w, i_{*}(y)\right)$ for $y \in H_{1}\left(M_{K}, \Lambda\right)$. We get a commutative diagram (cf. [Let00, cor. 2.9])

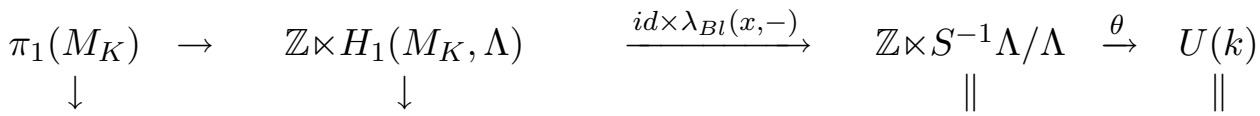

$$
\begin{aligned}
& \pi_{1}\left(N_{D}\right) \rightarrow \mathbb{Z} \ltimes \operatorname{Tor}_{\Lambda} H_{1}\left(N_{D}, \Lambda\right) \stackrel{i d \times \lambda_{B l, N_{D}}(w,-)}{\longrightarrow} \mathbb{Z} \ltimes S^{-1} \Lambda / \Lambda \stackrel{\theta}{\rightarrow} U(k) .
\end{aligned}
$$

This shows that $\theta \circ \alpha_{x}$ extends over $\pi_{1}\left(N_{D}\right)$. The first part of the theorem now follows from theorem 4.4 The second part follows from theorem 2.1 proposition 8.2 and the remark after theorem 8.4 . 


\subsection{More Examples}

In $\mathrm{Fr} 03 \mathrm{c}]$ we show that if for a knot the metabelian eta invariant ribbon obstruction vanishes, then all the abelian and metabelian sliceness obstructions vanish. The following example shows that furthermore the ribbon obstruction theorem is in fact stronger than these obstructions, considered as ribbonness obstructions.

Proposition 8.9 (Example 4) There exists a knot $S$ which is algebraically slice, (1.0)-solvable, which has zero STE-obstruction and zero metabelian $L^{2}$ eta invariant but which does not satisfy the condition for theorem 8.3, i.e. $S$ is not ribbon.

Proof Let $K$ be the ribbon knot of proposition 7.11 Recall that $\Delta_{K}(t)=$ $\Phi_{30}(t)^{2}$ and that $K$ has a unique metabolizer $P$. In particular $H_{1}\left(L_{K, k}\right)=0$ for all prime powers $k$ and a computation using lemma 2.2 shows that $\left|H_{1}\left(L_{K, 6}\right)\right|=$ 625 .

Now let $C$ be the knot of the proof of proposition 7.9 recall that $\int_{S^{1}} \sigma_{z}(C)=0$. Let $P_{6}:=\pi_{6}(P) \subset H_{1}\left(M_{K, 6}\right)$ be the projection of $P$. Let $A$ be a simple closed curve in $S^{3} \backslash K$, unknotted in $S^{3}$, such that $A \in \pi_{1}\left(S^{3} \backslash K\right)^{(1)}$, which lifts to a simple closed curve $\tilde{A}$ in the 6 -fold cover which presents a non-trivial element of order 5 in $H_{1}\left(M_{K, 6}\right) / P_{6}$.

We claim that the satellite knot $S:=S(K, C, A)$ satisfies the conditions stated in the proposition. The proof of proposition [7.11 shows that $S=S(K, C, A)$ is algebraically slice, is (1.0)-solvable, has zero STE-obstruction and zero metabelian $L^{2}$-eta invariant obstruction.

As remarked above, the Blanchfield pairing of $S$ has a unique metabolizer $P$. Let

$$
\chi: H_{1}\left(M_{K}, \Lambda\right) \rightarrow H_{1}\left(M_{K}, \Lambda\right) /\left(t^{6}-1\right) \rightarrow H_{1}\left(L_{S, 6}\right) \rightarrow S^{1}
$$

be a non-trivial character of order 5 , vanishing on $P_{6} \subset H_{1}\left(L_{S, 6}\right)$ such that $\chi(\tilde{A}) \neq 1 \in S^{1}$. By corollary 7.5

$$
\eta\left(M_{S}, \alpha_{(\chi, z)}^{S}\right)=\eta\left(M_{K}, \alpha_{(\chi, z)}^{K}\right)+\sum_{i=1}^{k} \eta\left(M_{C}, \alpha_{i}\right),
$$

where $\alpha_{i}$ denotes the representation $\pi_{1}\left(M_{C}\right) \rightarrow U(1)$ given by $g \mapsto \chi\left(\tilde{A}_{i}\right)^{\epsilon(g)}$. The first term is zero since $K$ is ribbon and $P$ is the unique metabolizer of the Blanchfield pairing (cf. theorem 8.3). The second term is non-zero since $\chi\left(\tilde{A}_{i}\right) \neq 1$ for at least one $i$ and by the properties of $C$. This shows that $\eta\left(M_{S}, \alpha_{(\chi, z)}^{S}\right) \neq 0$, i.e. $S$ is not ribbon by theorem 8.3 . 
Remark (1) The knot $S$ constructed in the proof has in fact the extra property that the Gilmer and the Letsche obstruction vanish. Indeed, in unpublished work we show that the Gilmer (respectively Letsche) obstruction is equivalent to the vanishing of certain eta invariants corresponding to irreducible prime power dimensional representations (respectively tensor products thereof). By construction the knot $S$ has no irreducible prime power dimensional representations.

(2) It is in fact possible to construct an example of a knot with all the above properties such that no multiple of $S$ is ribbon.

Note that it is not known whether the example given in the above proposition is slice or not.

Proposition 8.10 (Example 5) There exists a ribbon knot $S$ with the following property. There exists a prime power $k$ such that for all metabolizers $P$ we can find $\alpha \in P_{5}^{\text {irr,met }}\left(\pi_{1}\left(M_{K}\right)\right)$ with $\alpha(0 \times P)=0$ but such that $\eta\left(M_{K}, \alpha\right) \neq 0$.

Note that we do not restrict ourselves to representations $\alpha_{(z, \chi)}$, with $\chi$ of prime power order. The proposition shows that theorems 4.5 and 8.3 can't be strengthened to include all non prime power characters. The example shows that the set $P_{k}^{i r r, m e t}\left(\pi_{1}\left(M_{K}\right)\right)$ is in a sense maximal, i.e. that the prime power condition on the characters is indeed necessary.

Proof Consider $\Delta(t)=f(t) f\left(t^{-1}\right)$ where $f(t)=4-3 t+2 t^{2}+4 t^{3}-7 t^{4}+$ $t^{5}+2 t^{6}-3 t^{7}+t^{8}$. Terasaka [Te59] shows that any polynomial of the form $f(t) f\left(t^{-1}\right)$ can be realized by a slice knot. But this shows that $\Delta(t)$ can be realized by a metabolic Seifert matrix, hence there exists a ribbon knot $K$ with $\Delta_{K}(t)=\Delta(t)$. A computation shows that $H_{1}\left(L_{K, 5}\right)=1296=36^{2}$. Let $N$ be an integer greater than $\max \left\{\eta\left(M_{K}, \alpha\right) \mid \alpha \in R_{5}\left(\pi_{1}\left(M_{K}\right)\right)\right\}$. As in the proof of proposition 7.10 we see that such an $N$ exists.

Let $\tilde{A}_{1}, \ldots, \tilde{A}_{s} \in H_{1}\left(L_{K, 5}\right)$ be all elements. One can find simple closed curves $A_{1}, \ldots, A_{s} \subset S^{3} \backslash K$ such that $A_{i} \in \pi_{1}\left(S^{3} \backslash K\right)^{(1)}$ and such that for all $i=$ $1, \ldots, s$ the homology class $\tilde{A}_{i}$ is represented by one of the $k$ lifts of $A_{i}$ to $L_{k}$. Possibly after crossing changes of the representatives one can assume that $A_{1}, \ldots, A_{s}$ form in fact the unlink in $S^{3}$. Let $C$ be a ribbon knot with Seifert matrix $\oplus_{i=1}^{N+1} B_{1}$, and form the iterated satellite knot

$$
S:=S\left(K, C, \ldots, C, A_{1}, \ldots, A_{s}\right) .
$$

Note that $S$ is ribbon by proposition 7.1 . 
Let $P$ be a metabolizer for the Blanchfield pairing and let

$$
P_{5}:=\pi_{5}(P) \subset H_{1}\left(M_{K}, \Lambda\right) /\left(t^{5}-1\right)=H_{1}\left(L_{K, 5}\right) .
$$

Let $\chi: H_{1}\left(L_{5}\right) \rightarrow S^{1}$ be a non-trivial character of order 6 , vanishing on $P_{5}$. Then for all transcendental $z \in S^{1}$ we get

$$
\begin{aligned}
\eta\left(M_{S, 5}, \alpha_{(\chi, z)}^{S}\right) & =\eta\left(M_{K, 5}, \alpha_{(\chi, z)}^{K}\right)+\sum_{j=1}^{s} \sum_{i=1}^{5} \eta\left(M_{C_{j}}, \alpha_{i j}\right) \\
& \leq N+\sum_{j=1}^{s} \sum_{i=1}^{5}(N+1) \sigma_{\chi\left(\left(\tilde{A}_{j}\right)_{i}\right)}\left(B_{1}\right) .
\end{aligned}
$$

Since $\chi$ is of order 6 and by construction of $A_{1}, \ldots, A_{s}$, we can find $(i, j)$ such that $\chi\left(\left(\tilde{A}_{j}\right)_{i}\right)=e^{2 \pi i / 6}$, but recall that $\sigma_{z}\left(B_{1}\right)=0$ for all $z$ except for $z=e^{2 \pi i / 6}, e^{2 \pi 5 i / 6}$ where $\sigma_{z}\left(B_{1}\right)=-1$. This shows that $\eta\left(M_{S}, \alpha_{(\chi, z)}^{S}\right) \leq N+$ $(N+1)(-1)=-1$.

Remark The degree of the Alexander polynomial of $K$ is quite large, but it was the polynomial of lowest degree we could find with $\left|H_{1}\left(L_{k}\right)\right|$ being divisible by 6 for some prime power $k$.

\section{References}

[A76] M. Atiyah, Elliptic operators, discrete groups and von Neumann algebras, Colloque "Analyse et Topologie" en l'Honneur de Henri Cartan (Orsay, 1974), Asterisque, No. 32-33, Soc. Math. France, Paris: 43-72 (1976) MathReview

[APS75] M. Atiyah, V. Patodi, I. Singer, Spectral asymmetry and Riemannian geometry II, Math. Proc. Camb. Phil. Soc, 78: 405-432 (1975) MathReview

[B57] R. Blanchfield, Intersection theory of manifolds with operators with applications to knot theory, Ann. of Math. (2) 65: 340-356 (1957) MathReview

[CG78] A. Casson, C. Gordon, On slice knots in dimension three, Algebraic and geometric topology (Proc. Sympos. Pure Math., Stanford Univ., Stanford, Calif., 1976), Part 2, 39-53, Proc. Sympos. Pure Math., XXXII, Amer. Math. Soc., Providence, R.I. (1978) MathReview

[CG86] A. Casson, C. Gordon, Cobordism of classical knots, Progr. Math., 62, Á la recherche de la topologie perdue, 181-199, Birkhäuser Boston, Boston, MA (1986) MathReview

[ChG85] J. Cheeger, M. Gromov, Bounds on the von Neumann dimension of $L^{2}$ cohomology and the Gauss-Bonnet theorem for open manifolds, J. Differential Geom. 21, no. 1: 1-34 (1985) MathReview

[COT03] T. Cochran, K. Orr, P. Teichner, Knot concordance, Whitney towers and $L^{2}$-signatures, Ann. of Math. (2) 157, no. 2: 433-519 (2003) MathReview 
[COT04] T. Cochran, K. Orr, P. Teichner, Structure in the classical knot concordance group, Comment. Math. Helv. 79, no. 1:105-123 (2004) MathReview

[CF64] P. E. Conner, E. E. Floyd, Differentiable periodic maps, Ergebnisse der Mathematik und ihrer Grenzgebiete, N. F., Band 33, Springer-Verlag (1964) MathReview

[Fox61] R. H. Fox, Some problems in knot theory, 1962 Topology of 3-manifolds and related topics (Proc. The Univ. of Georgia Institute): 168-176 (1961) MathReview

[FQ90] M. Freedman, F. Quinn, Topology of 4-manifolds, Princeton Mathematical Series, 39. Princeton University Press, Princeton, NJ (1990) MathReview

[Fr03] S. Friedl, Full signature invariants for $L_{0}(F(t))$, Preprint (2003), to appear in Proc. Amer. Math. Soc.

[Fr03b] S. Friedl, Link concordance, boundary link concordance and eta-invariants, Preprint (2003), to appear in Math. Proc. Camb. Phil. Soc.

[Fr03c] S. Friedl, $L^{2}$-eta invariants and their approximation by unitary eta invariants, Preprint (2003), to appear in Math. Proc. Camb. Phil. Soc.

[Fr03d] S. Friedl, Eta invariants as sliceness obstructions and their relation to CassonGordon invariants, Thesis, Brandeis University (2003)

[Gi83] P. Gilmer, Slice knots in $S^{3}$, Quart. J. Math. Oxford Ser. (2) 34, no. 135: 305-322 (1983) MathReview

[Gi93] P. Gilmer, Classical knot and link concordance, Comment. Math. Helv. 68, no. 1: 1-19 (1993) MathReview

[Gom86] R. Gompf, Smooth concordance of topologically slice knots, Topology 25, no. 3: 353-373 (1986) MathReview

[Gor77] C. McA. Gordon, Some aspects of classical knot theory. Knot theory (Proc. Sem., Plans-sur-Bex, 1977), Lecture Notes in Math. 685:1-65 (1978) MathReview

[Gor81] C. McA. Gordon, Ribbon concordance of knots in the 3-sphere, Math. Ann. 257, no. 2: 157-170 (1981) MathReview

[HS71] P. Hilton, U. Stammbach, A course in homological algebra, Springer Verlag, Berlin and New York (1971) MathReview

[J81] B. Jiang, A simple proof that the concordance group of algebraically slice knots is infinitely generated, Proc. Amer. Math. Soc. 83: 189-192 (1981) MathReview

[Kea73] C. Kearton, Classification of simple knots by Blanchfield duality, Bull. Amer. Math. Soc. 79: 952-955 (1973) MathReview

[Kea75] C. Kearton, Presentations of n-knots, Trans. Amer. Math. Soc. 202: 123-140 (1975) MathReview

[Kea75b] C. Kearton, Cobordism of knots and Blanchfield duality, J. London Math. Soc. (2) 10, no. 4: 406-408 (1975) MathReview 
[Ker65] M. Kervaire, On higher dimensional knots, 1965 Differential and Combinatorial Topology, Princeton Univ. Press, Princeton, N.J.: pp. 105-119 (1965) MathReview

[Kim02] T. Kim, Filtration of the classical knot concordance group and Casson-Gordon invariants, Preprint (2002), to appear in Math. Proc. Cambridge Philos. Soc.

[Kim03] T. Kim, Obstructions to slicing and doubly slicing knots, Thesis, Indiana University, Bloomington (2003)

[Kim04] T. Kim, New obstructions to doubly slicing knots, Preprint (2004)

[La93] S. Lang, Algebra, Revised third edition. Graduate Texts in Mathematics, 211. Springer-Verlag, New York (2002) MathReview

[Let95] C. Letsche, An obstruction to slicing knots using the eta invariant, Thesis, Indiana University, Bloomington (1995)

[Let00] C. Letsche, An obstruction to slicing knots using the eta invariant, Math. Proc. Cambridge Phil. Soc. 128, no. 2: 301-319 (2000) MathReview

[Lev69] J. Levine, Knot cobordism groups in codimension two, Commentarii Mathematici Helvetici 44: 229-244 (1969) MathReview

[Lev69b] J. Levine, Invariants of knot cobordism, Inventiones Mathematicae 8: 98-110 (1969) MathReview

[Lev70] J. Levine, An algebraic classification of some knots of codimension two, Comment. Math. Helv. 45: 185-198 (1970) MathReview

[Lev77] J. Levine, Knot modules. I, Trans. Amer. Math. Soc. 229: 1-50 (1977) MathReview

[Lev89] J. Levine, Metabolic and hyperbolic forms from knot theory, J. Pure Appl. Algebra 58, no. 3: 251-260 (1989) MathReview

[Lev94] J. Levine, Links invariants via the eta invariant, Commentarii Mathematici Helvetici 69: 82-119 (1994) MathReview

[Lit84] R. Litherland, Cobordism of satellite knots, Four-manifold theory (Durham, N.H., 1982), Contemp. Math. 35, Amer. Math. Soc., Providence, RI: 327-362 (1984) MathReview

[Liv02] C. Livingston, Seifert forms and concordance, Geom. Topol. 6: 403-408 (2002) paper 14 MathReview

[LS01] W. Lück, T. Schick, Approximating $L^{2}$-signatures by their compact analogues, Preprint (2001)

[LS03] W. Lück, T. Schick, Various $L^{2}$-signatures and a topological $L^{2}$-signature theorem, High-dimensional manifold topology: 362-399, Word Sci. Publishing, River Edge, NJ (2003) MathReview

[Ma77] T. Matumoto, On the signature invariants of a non-singular complex sesquilinear form, J. Math. Soc. Japan 29, no. 1: 67-71 (1977) MathReview 
[Mu65] K. Murasugi, On a certain numerical invariant of link types, Trans. Amer. Math. Soc. 117: 387-422 (1965) MathReview

[Ra96] A. Ranicki, On the Hauptvermutung, The Hauptvermutung book, 3-31, $K$ Monogr. Math., 1, Kluwer Acad. Publ., Dordrecht (1996) MathReview

[Ra98] A. Ranicki, High-dimensional knot theory, Springer Monographs in Mathematics, Springer-Verlag, New York (1998) MathReview

[Ru83] D. Ruberman, Doubly slice knots and the Casson-Gordon invariants, Trans. Amer. Math. Soc. 279, no. 2: 569-588 (1983) MathReview

[S71] D. W. Sumners, Invertible knot cobordisms, Comment. Math. Helv. 46: 240-256 (1971) MathReview

[Te59] H. Terasaka, On null-equivalent knots, Osaka Math. J. 11: 95-113 (1959) MathReview

[Tr73] H. F. Trotter, On S-equivalence of Seifert matrices, Invent. Math. 20: 173-207 (1973) MathReview

Department of Mathematics, Rice University, Houston, TX 77005, USA

Email: friedl@rice.edu

URL: http://math.rice.edu/ ${ }^{\sim}$ riedl/

Received: 17 January 2004 Revised: 13 September 2004 